\title{
Large eddy simulation on the effects of pressure on syngas/air turbulent nonpremixed jet flames
}

Pietro P. Ciottolia ${ }^{a *}$, Bok Jik Lee ${ }^{\mathrm{b}}$, Pasquale E. Lapenna ${ }^{\mathrm{a}}$, Riccardo Malpica Galassi ${ }^{\mathrm{a}}$, Francisco E. Hernández Pérez ${ }^{\mathrm{c}}$, Emanuele Martelli ${ }^{\mathrm{d}}$, Mauro Valorani ${ }^{\mathrm{a}}$, Hong G. $\mathrm{Im}^{\mathrm{c}}$

${ }^{\text {a }}$ Sapienza University of Rome, Rome, Italy

${ }^{\mathrm{b}}$ Department of Mechanical and Aerospace Engineering, Seoul National University, Seoul, South Korea

${ }^{\mathrm{c}}$ Clean Combustion Research Center, KAUST, Saudi Arabia

${ }^{\mathrm{d}}$ Campania University L. Vanvitelli, Aversa, Italy

\begin{abstract}
The influence of increasing pressure on nonpremixed syngas/air turbulent jet flames is numerically investigated using large eddy simulations in conjunction with a steady laminar flamelet approach. The applicability of the steady flamelet approach is assessed through an extensive parametric study of laminar counterflow flames and tangential stretching rate analysis on target flame structures at different pressures. Two set of large eddy simulations, exploring pressure values up to $10 \mathrm{~atm}$, are carried out. The first one (series A) is characterized by a constant jet Reynolds number, while the second one (series B) is characterized by a constant jet inlet velocity. Both campaigns show narrower flame brushes and reduced radical concentrations with increasing pressure. While for series A the flame length is not sensitive to pressure, a longer flame brush is noticed for series $\mathrm{B}$, being mainly caused by the increased mass flow rate. The sensitivity of the local flame behavior to pressure, such as the $\mathrm{OH}$ layer thickness and position, is compared to the available experimental results, showing similar trends with a satisfactory agreement.

Keywords: Large Eddy Simulation, Turbulent jet flames, Pressure effects, High-pressure combustion
\end{abstract}

\footnotetext{
*Corresponding author

Email address: pietropaolo.ciottoli@uniroma1.it (Pietro P. Ciottolia*)
} 


\section{Introduction}

To achieve optimal efficiencies and reduced sizes, practical combustion devices such as gas turbines, reciprocating engines and rocket engines operate at elevated pressure and in a highly turbulent environment. It is known that pressure increases combustion intensity, in terms of energy released per unit volume, which scales with the square of pressure. This results in a higher energy output of the device as well as an overall higher system efficiency. While high pressure combustion devices are relatively mature technologies and have been successfully utilized for decades, the complex interplay of various phenomena occurring in such devices is not fully understood due in part to the difficulties in obtaining systematic and detailed information by experiments or simulations.

In the context of turbulent nonpremixed jet flames, reliable experimental measurements of spatially and temporally resolved species concentrations as well as temperature have been obtained mostly at atmospheric pressure [1-3]. High pressure investigations have been limited to laminar flame configurations, mainly aimed at gaining insights into soot production and oxidation [17, 18]. Computational studies of high pressure nonpremixed combustion have also been mainly conducted in laminar flames, either in the counterflow configuration [35, 50, 52] or by the flamelet formulation in the mixture fraction space [26, 27].

To bridge the gap and gain insights into turbulent combustion dynamics at high pressures, new experimental facilities have been established at King Abdullah University of Science and Technology (KAUST) [10, 41, 57] and laser diagnostic measurements have been conducted to characterize the key flame observables for syngas/air jet flames by Boyette et al. [5]. In this study, low-speed OH-PLIF measurements were undertaken to capture the flame structure in a small window placed between 5 and 8.2 diameters downstream the injector. This was the first of its kind to visualize the sensitivity of the $\mathrm{OH}$ layer to pressure, both in the case of constant inflow velocity and constant Reynolds number.

The present paper is the first report of the corresponding computational simulations based on the large eddy simulation (LES) framework for the same configurations in order to provide detailed spatial information for comparison. The LES formulation features a standard steady 
laminar flamelet (SLF) model [45, 47], which is validated at atmospheric pressure against the Sandia/ETH-Zurich $\mathrm{CO} / \mathrm{H}_{2} / \mathrm{N}_{2}$ (Flame A) database of measurements [1]. This flame has already been selected as a benchmark for a comparative study of the steady and unsteady flamelet LES approaches [22], and the assessment of one dimensional turbulence (ODT) chemical closure model capabilities [13]. Moreover, the same flame configuration was numerically investigated using a conditional moment closure (CMC) and an unsteady flamelet model [21], an Eulerian particle flamelet model (EPFM) [23], a fractal model (FM) [12], and a transported probability density function (PDF) approach [65].

In the present paper, two LES campaigns of parametric studies are carried out, each consisting of three cases with pressure values of 2, 5, and $10 \mathrm{~atm}$, consistent with the experimental counterpart [5]. In the first campaign, referred to as series $A$, the inflow velocity is varied with pressure in order to keep the inflow Reynolds number constant, while in the second campaign, referred to as series $\mathrm{B}$, the inflow velocity is kept constant. To assess the effects of pressure on laminar nonpremixed syngas/air flames and the applicability of the SLF model to a turbulent jet flame at high pressures, the impact of pressure on spatial and time scales is investigated using the classical counterflow configuration [19]. The effects of pressure on the time scales of laminar nonpremixed flames are also examined by using the computational singular perturbation (CSP) analysis [24, 37, 60], in particular in the context of the new tangential stretching rate (TSR) concept [38-40, 59, 61].

The chemical kinetic scheme employed in all the computations is a 12-species, 33-reactions, comprehensive $\mathrm{CO} / \mathrm{H}_{2} / \mathrm{O}_{2} / \mathrm{N}_{2}$ detailed mechanism [33], hierarchically built upon the hydrogen mechanism in [34], and validated against a wide range of experimental conditions in the pressure range $0.15-9.6 \mathrm{~atm}$, thus adequately covering the parametric conditions under study.

The paper is organized as follows. The applicability of the SLF model for increasing pressure is assessed in Section 2. The LES-SLF computational framework is described and validated against the Sandia/ETH-Zurich Flame A experimental database in Section 3. The effects of pressure on turbulent jet flames are discussed in sec. 4, for both series A and B, highlighting the similarities and differences. Global flame behaviors and comparison with the experimental results [6] are presented in sec.6. Finally, the results are summarized with concluding remarks in Section 7 . 


\section{Sensitivity of laminar nonpremixed flame structure to pressure}

Since its introduction in [44], many extensions of the SLF method have been proposed, validated and employed in turbulent non-premixed flames in a wide range of conditions. In particular, many formulations have overcome the structural drawbacks of the SLF method, accounting for unsteady effects on the flame structure such as auto-ignition and local quenching, as well as other conditions as partial premixing. These include the flamelet progress variable (FPV) [14, 46], the unsteady laminar flamelet (ULF) [47, 48], the unsteady flamelet progress variable (UFPV) [15], and unstrained premixed flamelets with mixture stratification and presumed joint probability density function [7, 8, 25, 54]. On the other hand, it is well known that the SLF approach remains valid if the chemical time scales are sufficiently faster than the transport/residence time scales, so that such unsteady effects on the flame structure can be safely neglected, i.e. under elevated Damkhöler number conditions [43, 45]. As a baseline study, the pressure effects on laminar counter-flow flames is examined in terms of the fluid dynamic and chemical time and length scales. This analysis is based on steady-state solutions of strained opposed-flow laminar flames and aimed at assessing the validity of the SLF combustion closure for LES of turbulent diffusive flames at increased pressures. The CSP/TSR analysis allows a systematic identification of the characteristic chemical timescales to assess the effects of pressure on the Damkhöler number.

\subsection{Fluid dynamic timescales sensitivity to pressure}

The sensitivity of the fluid dynamic space and time scales to pressure is analyzed by two parametric studies on counterflow flames, consistent with the LES campaigns to be studied. In the first set, the mass flow rate are kept constant for both fuel and air streams, whereas in the second one the velocity is kept constant. The OPPDIF code [36] is employed for the simulations, in which the fuel and oxidizer velocities are prescribed as boundary conditions, and the strain rate $a$ and the scalar dissipation rate $\chi$ are calculated as part of the solutions.

An atmospheric reference condition is chosen following the Sandia/ETH-Zurich Flame A [1], with $Z_{\text {stoi }}=0.295$ and the fuel and oxidizer velocities set to obtain an intermediate strain value $a \approx 25 s^{-1}$. The solutions are obtained using detailed transport, with mixture averaged diffusivities.Results are shown in terms of temperature, $\mathrm{OH}$ radical, mixture fraction $Z$, and scalar 
dissipation rate $\chi$, with $Z$ defined according to Bilger's definition [4].

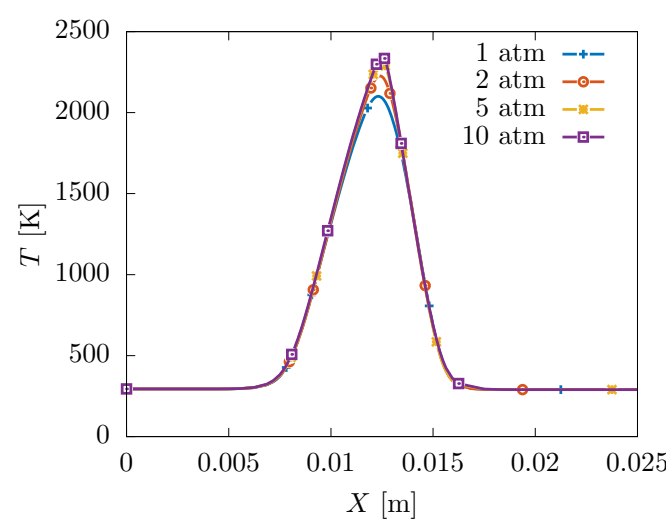

(a) Temperature

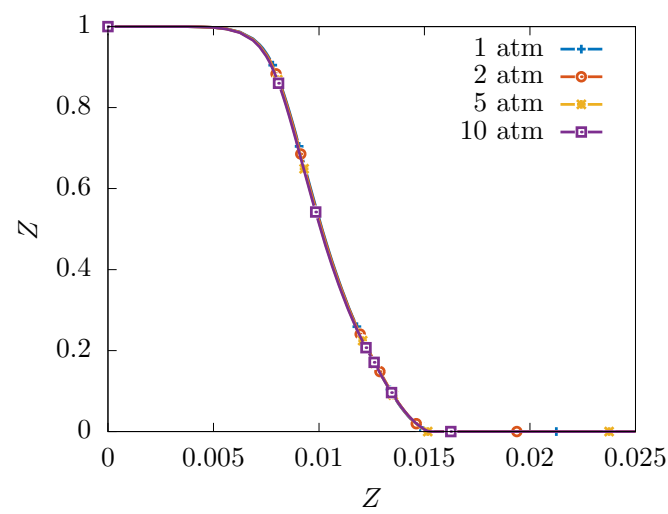

(c) Mixture fraction

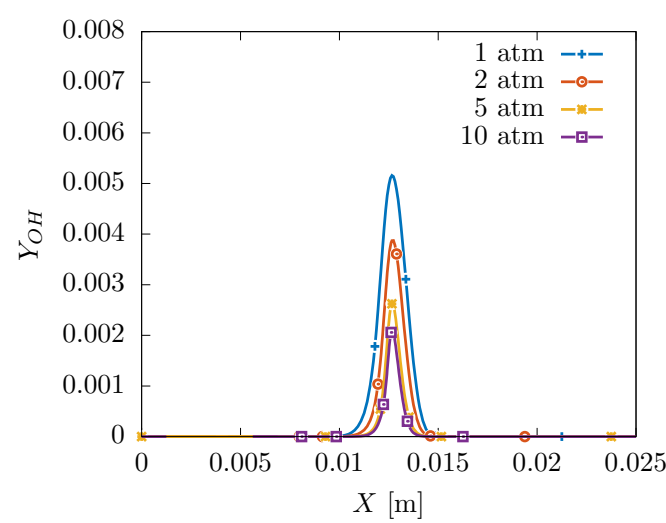

(b) $\mathrm{OH}$ mass fraction

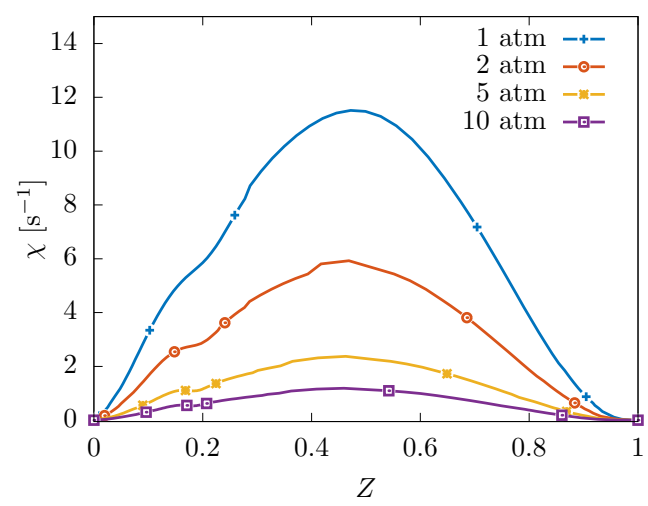

(d) Scalar dissipation rate

Figure 1: Laminar counterflow solutions with constant mass flow rate: spatial distributions of temperature, $\mathrm{OH}$ mass fraction, mixture fraction, and scalar dissipation rate for pressure values $p=1,2,5$ and $10 \mathrm{~atm}$.

Figure 1 shows the results of the first series, which is performed by linearly diminishing the inflow velocities with pressure to compensate for the density increase and ensure a constant mass flow rate for all the computations, consistently with the LES series A campaign. The temperature spatial distribution is shown in Fig. 1a, where an increase of maximum temperature is observed in the stoichiometric region with increasing pressure, indicating that the chemical reaction reaches the equilibrium with increased recombinations. The spatial distribution of $\mathrm{OH}$ mass fraction is plotted in Fig. 1b, showing a decrease in the $\mathrm{OH}$ radical concentration and flame thickness. On the other hand, the mixture fraction distribution shown in Fig. 1d appears to be unaffected by the 
pressure rise, confirming the laminar flame theory that the diffusive layer scales with the mass flow rate [49]. The profiles of scalar dissipation rate $\chi=D_{Z}|\nabla(z)|^{2}$, computed by assuming $D_{Z}$ equal to the mixture thermal diffusivity, are shown in Fig. 1d. The $\chi$ profiles in mixture fraction space remain qualitatively unchanged with pressure, which is expected since the mass diffusivity is inversely proportional to density, leading to a scalar dissipation rate decrease inversely proportional to pressure $\left(\chi \propto p^{-1}\right)$.

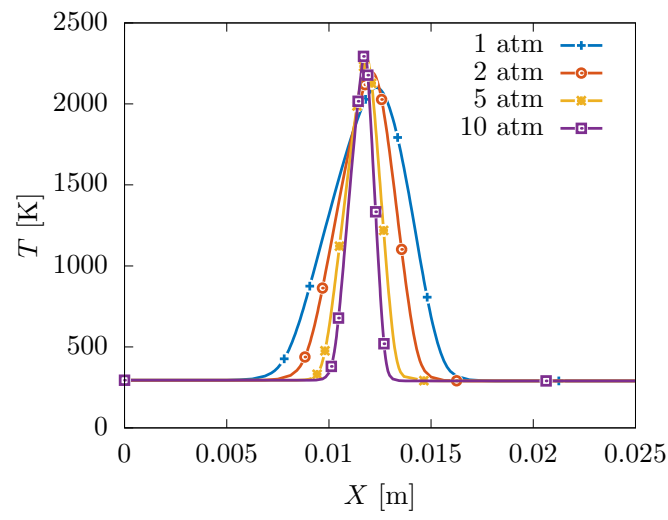

(a) Temperature

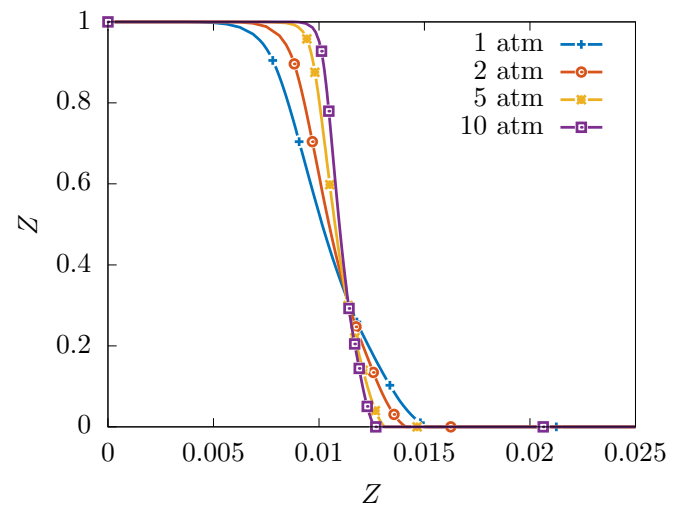

(c) Mixture fraction

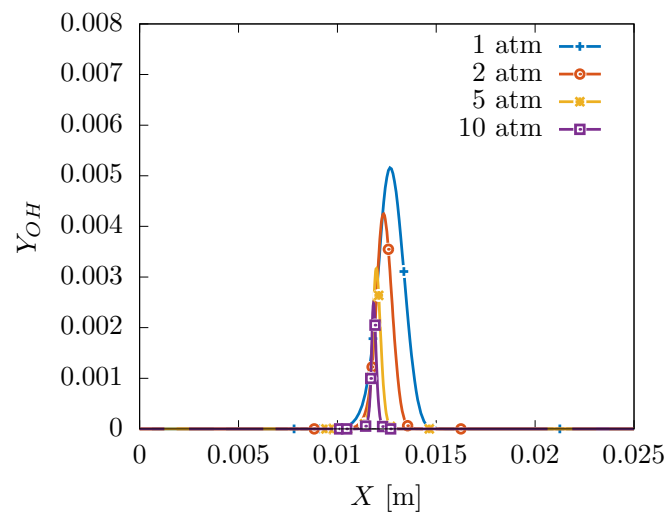

(b) $\mathrm{OH}$ mass fraction

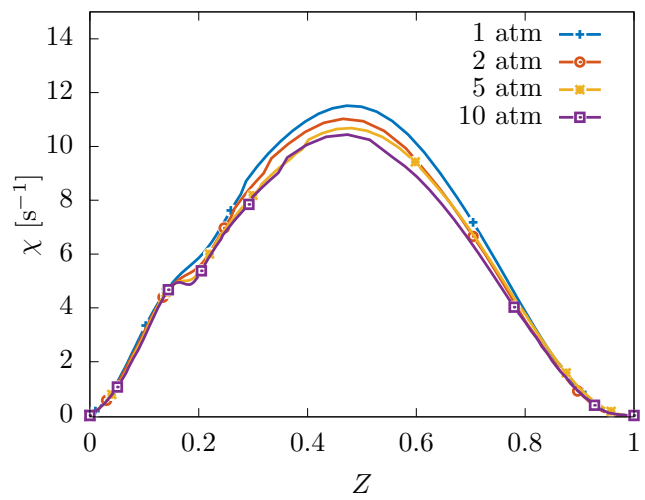

(d) Scalar dissipation rate

Figure 2: Laminar counterflow solutions with constant jet streams velocity: the spatial distribution of temperature, $\mathrm{OH}$ mass fraction, mixture fraction, and scalar dissipation rate for pressure values $p=1,2,5$ and $10 \mathrm{~atm}$.

Figure 2 shows the results of the second series at fixed fuel and oxidizer stream velocity, consistent with the LES series B campaign. As the pressure increases, the linear increase in density leads to a proportional increase in both fuel and oxidizer mass flow rates. The temperature and $\mathrm{OH}$ 
radical mass fraction spatial distributions are displayed in Fig. $2 \mathrm{a}$ and $2 \mathrm{~b}$, respectively. The trend is similar to the one observed for the constant mass flow rate campaign with increasing pressure. Nonetheless, unlike the first series, the mixture fraction spatial distribution is much more sensitive to pressure as seen in Fig. 2c. This change in the $Z$ distribution highlights that the diffusive layer becomes narrower because of the increase in the mass flow rates. The narrowing of the diffusive region implies a shift in the stoichiometric mixture fraction as well as a shift in the flame position, which are noticeable in the $T$ and $\mathrm{OH}$ distributions. The profiles of $\chi$ in mixture fraction space are displayed in Fig. 2d, showing a weak dependency on the pressure increase, due to the increase in the $Z$ gradients that partially compensate for the linear decrease in the molecular diffusivity.

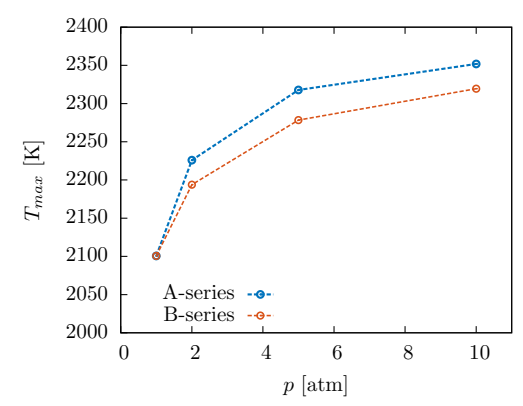

(a) Temperature

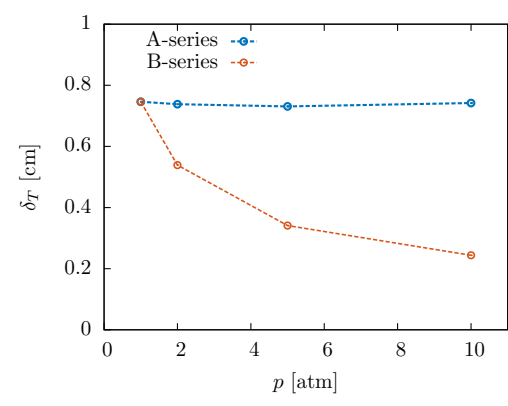

(b) Flame thickness

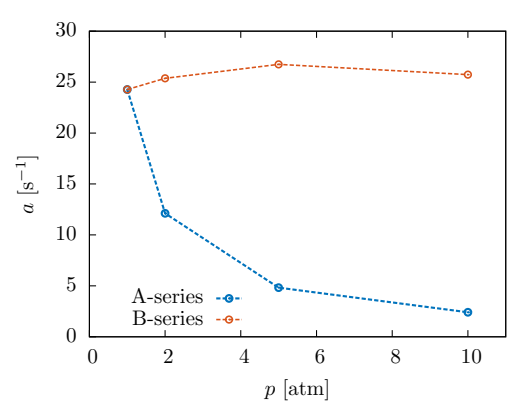

(c) Strain rate

Figure 3: Sensitivity of temperature T, flame thickness $\delta_{T}$, and strain rate $a$ for the constant inflow velocity set (series A) and the constant mass flow rate set (series B), for $p=1,2,5$ and $10 \mathrm{~atm}$.

A comparison of the two series are shown in Fig. 3 in terms of maximum flame temperature $T_{\max }$, flame thickness $\delta_{T}$, and strain rate $a$, as defined in [19, 36]. An increase in $T_{\max }$ is shown for both cases while $\delta_{T}$ is found to be almost insensitive to pressure in case of constant mass flow rate (series A), while it decreases in the case of a constant inflow velocity (series B). The opposite behavior is observed for $a$, as shown in Fig. 3c, where a pressure increase leads to a pronounced strain rate decrease if mass flow rate is kept constant (series A), and to a negligible increase if the inflow velocity is kept constant (series B). The principal effect of pressure on the chemical source terms is to push the composition through the fully burned state, with a decrease in radicals concentrations and an increase in temperature, the latter being more pronounced when the mass flow rate is kept constant because of the concomitant decrease in the strain rate. A summary 
of pressure effects on both $T_{\max }$ and $\delta_{T}$ is reported in Fig. 4 for a wider range of strain rates confirming that higher pressures lead to more robust flames, which are less sensitive to strain rate perturbations.

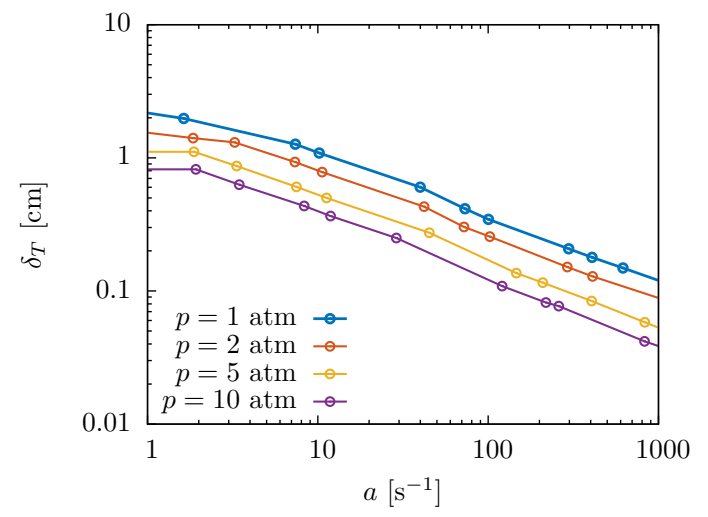

(a) Flame thickness

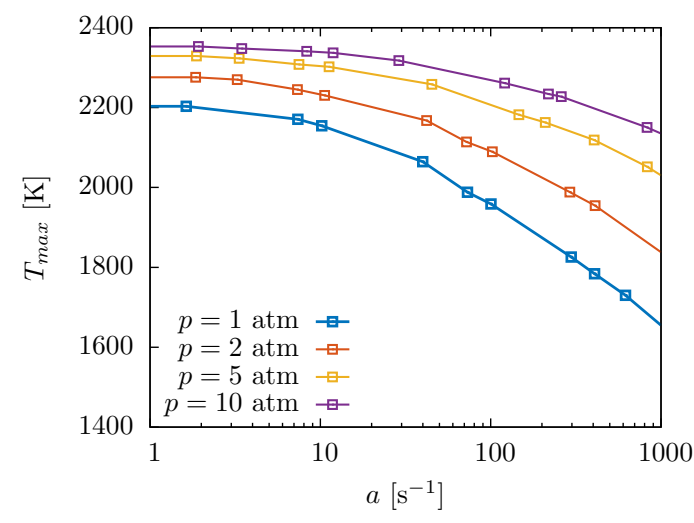

(b) Temperature

Figure 4: Sensitivity of flame thickness $\delta_{T}$ and maximum temperature $T_{\max }$ to the strain rate $a$ for pressures $p=1,2$, 5 and $10 \mathrm{~atm}$.

\subsection{Chemical timescales sensitivity to pressure}

For nonpremixed combustion, the Damkhöler number is commonly based on a diffusion time scale associated with the scalar dissipation rate $\chi$ and a chemical time scale $\tau_{c}$, and expressed as $D a=1 /\left(\tau_{c} \chi\right)$. Identifying the characteristic $\tau_{c}$ is not trivial for a combustion system with detailed chemistry. In this study, a rigorous definition of $\tau_{c}$ is provided within the computational singular perturbation (CSP) framework, through the tangential stretching rate (TSR) extension to reactiondiffusion systems (extended TSR). While a detailed description of TSR and extended TSR can be found in [38, 59, 61], it is worth to recall that the TSR and its extension to systems of partial differential equations (pde), namely $\omega_{\tilde{\tau}}$ and $\omega_{\tilde{\tau}_{p d e}}$, are linear combinations of the chemical system eigenvalues, where the weights are proportional to the energy contained in each associated mode amplitude.

Since fast/exhausted modes have negligible amplitude by construction [24, 62], the energy of the system is stored in the slow modes, among which only a few contribute substantially to 
the system's evolution [59]. These are the modes identified by the TSR, which belong to the active subspace. The other slow modes, typically slower than the active ones, give negligible contribution to the species evolution [63]. Hence, the TSR represents the most energetic scale at which the system evolves, and is a rational candidate for the estimation of a chemical timescale $\tau_{c}$. Indeed, the TSR has the physical dimension of the reciprocal of a timescale, and, in a purely reactive system, it represents the stretch rate induced by the chemical source term on a line element advected by two adjacent trajectories.

Furthermore, in a reactive-diffusive system, the extended TSR, $\omega_{\tau, p d e}$, is the stretching rate along the unit vector aligned with the state evolution direction due to the full reactive-diffusive system, while the TSR, $\omega_{\tau}$, is the same quantity in the direction of the chemical source terms only, i.e. the direction that the state would follow if the diffusive terms were abruptly set to zero. In other words, the $\omega_{\tau}$ can be seen as the timescale that the chemical source term would exhibit to recover the chemical equilibrium prevented by the diffusive term. Moreover, when the diffusive term importance is negligible with respect to the chemical source term, the two TSRs coincide, highlighting the regions which are dominated by chemistry. Here $\omega_{\tau, p d e}$ is employed to estimate the chemical timescale $\tau_{c}=1 / \omega_{\tau, p d e}$, while the regions where $\omega_{\tau}$ and $\omega_{\tau, p d e}$ differ are characterized by a diffusion-dominated dynamics. Hence, the extent of the region where $\omega_{\tau}$ and $\omega_{\tau, p d e}$ coincide represents an accurate measure of the reactive layer thickness.

The present a priori analysis is carried out employing steady state flamelet solutions with $\chi=20 \mathrm{~s}^{-1}$, and for pressure values of $p=1,2,5$ and $10 \mathrm{~atm}$. These solutions have been obtained by means of the flamelet solver [9] available in the CSPTk package [58], which integrates in time the unsteady flamelet equations to the steady state, using DVODE and handles the thermokinetic databases via the TChem package [55]. Equal diffusivity is assumed, since the conditional mean compositional structure of the syngas turbulent flame considered in the present work is represented with good accuracy by equal diffusivity laminar flame structures [1]. The results of the CSP analysis are shown in Fig. 5a where, for each pressure value, the temperature profiles, the maximum eigenvalue associated with the chemical source term, representing the reciprocal of the fastest timescale, and both $\omega_{\tau}$ and $\omega_{\tau, p d e}$ are all displayed. The CSP analysis reveals that a pressure increase leads to higher temperatures and faster timescales, as well as a shrinkage 


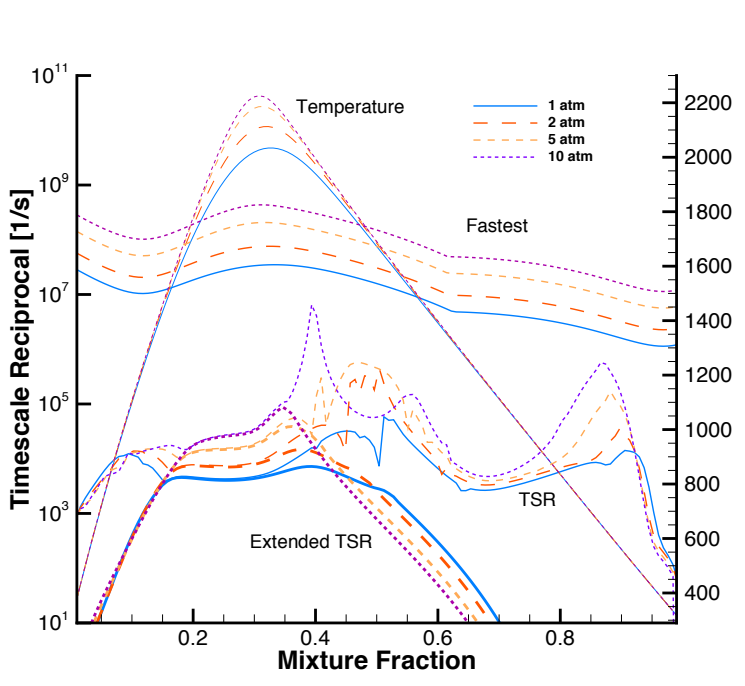

(a)

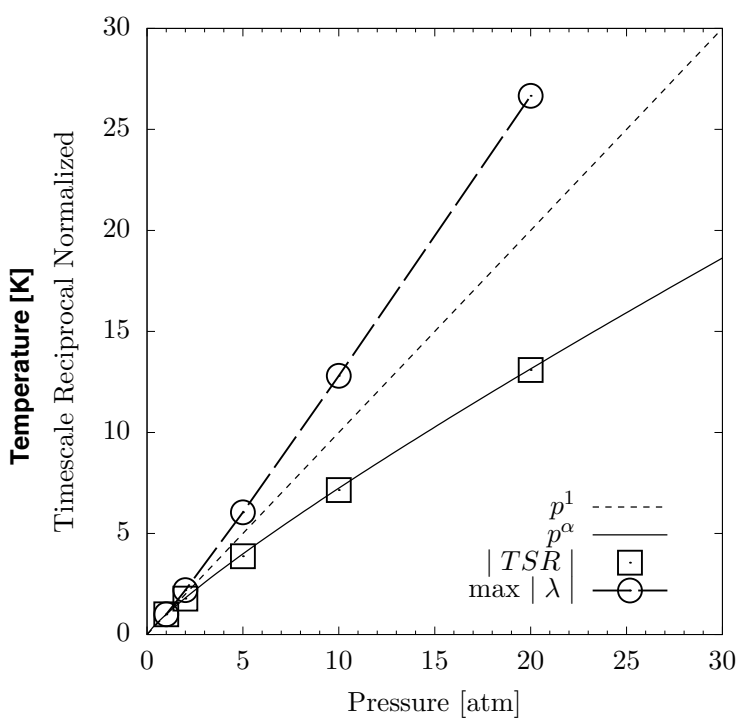

(b)

Figure 5: TSR/CSP analysis on pressure dependence of chemical timescales for pressure $p=1,2,5$ and 10 atm. (a) Temperature, TSR, extended TSR and reciprocal of the fastest time scale. (b) Chemical timescales sensitivity to pressure.

of the region where the system dynamics is governed by chemical kinetics, i.e. where $\omega_{\tau}$ and $\omega_{\tau, p d e}$ coincide. Moreover, the observation of the timescale values at the stoichiometric mixture fraction $(Z=0.295)$ reveals that the reciprocal of the fastest time scale $|\lambda|$ increases more than linearly with pressure, while the most energetic chemical timescale $\tau_{c}=1 / \omega_{\tau, p d e}$ is such that: $\tau_{c} / \tau_{c, 1 a t m}=\left(p / p_{1 a t m}\right)^{\alpha}$, with $\alpha \approx 0.86$, as shown in Fig. $5 \mathrm{~b}$

\subsection{A priori assessment of SLF-LES applicability}

To summarize the basic laminar flame behavior, if the fuel and oxidizer streams velocity is kept constant, the scalar dissipation rate is weakly sensitive to pressure, mostly because the decrease in molecular diffusivity is offset by an increase in the mixture fraction gradients. On the other hand, in the case of constant mass flow rate, the mixture fraction spatial distribution remains insensitive to pressure, and the scalar dissipation rate reduces with pressure. From the CSP/TSR analysis, it is concluded that an increase in pressure leads to a sub-linear chemical time scale increase for a fixed scalar dissipation rate, thus contributing to an overall increase in the characteristic Damkhöler 
number of the system. This trends, together with the experimental evidence at ambient pressure of a fully anchored flame not experiencing any local quenching, suggest that the SLF approach remains valid for LES of syngas/air turbulent nonpremixed jet flames at elevated pressures.

\section{LES Implementation and Validation}

The effects of a pressure increase on turbulent jet flames is now studied by LES with SLF approach as the combustion closure. The numerical setup and its validation against available experimental data are described in the present section.

\subsection{Numerical setup}

The LES studies are carried out using flameletFoam [42], a finite-volume solver based on the computational fluid dynamics (CFD) OpenFoam framework [64], which employs the standard SLF approach for the subgrid-scale combustion closure [45]. The solver uses a pressure-based, time-implicit segregated algorithm to integrate the filtered momentum, pressure, and energy equations using second-order spatial discretization. The steady state laminar solutions used for the tabulation have been obtained using the unsteady flamelet solver [9] with the chemical mechanism described in the previous section. As commonly done in flamelet approaches [49], these laminar solutions are firstly obtained at different values of the scalar dissipation rate of the mixture fraction at stoichiometric conditions, and secondly the filtered scalar variables are reconstructed by integrating the flamelet solutions with a presumed beta PDF for the mixture fraction and a Dirac delta for the scalar dissipation rate. Thermochemical properties, such as sensible enthalpy and species mass fractions are then extracted from the flamelet look-up tables using representative parameters, i.e. the filtered mixture fraction $\widetilde{Z}(x, t)$, the subgrid scale (SGS) mixture fraction variance $\widetilde{Z^{\prime \prime 2}}(x, t)$, and scalar dissipation rate $\widetilde{\chi}(x, t)$. These parameters allow to account for the effects of the fluid-dynamic strain rate on the flame structure as well as the local degree of mixing, thus providing a consistent averaging of each thermochemical properties taking into account SGS scalar fluctuations [30, 31]. The filtered mixture fraction and its variance are evolved in time $(t)$ 
and space $\left(x_{i}\right)$ according to the modeled transport equations:

$$
\begin{gathered}
\frac{\partial(\bar{\rho} \widetilde{Z})}{\partial t}+\frac{\partial\left(\bar{\rho} \widetilde{u_{i}} \widetilde{Z}\right)}{\partial x_{i}}=\frac{\partial}{\partial x_{i}}\left[\bar{\rho}\left(D_{z}+D_{t}\right) \frac{\partial \widetilde{Z}}{\partial x_{i}}\right] \\
\frac{\partial\left(\bar{\rho} \widetilde{Z^{\prime \prime 2}}\right)}{\partial t}+\frac{\partial\left(\bar{\rho} \widetilde{u_{i}} \widetilde{Z^{\prime \prime 2}}\right)}{\partial x_{i}}=\frac{\partial}{\partial x_{i}}\left[\bar{\rho}\left(D_{z}+D_{t}\right) \frac{\partial \widetilde{Z^{\prime \prime 2}}}{\partial x_{i}}\right]+ \\
+2 \bar{\rho}\left(D_{z}+D_{t}\right) \frac{\partial \widetilde{Z}}{\partial x_{i}} \frac{\partial \widetilde{Z}}{\partial x_{i}}-2 \widetilde{\rho \chi}
\end{gathered}
$$

where $D_{z}$ is the molecular diffusivity of the mixture fraction, assumed to be equal to the thermal diffusivity (Le=1), and $D_{t}$ is the turbulent diffusivity, modeled according to the relation $S c=$ $\mu_{t} /\left(\bar{\rho} D_{t}\right)=0.7$. The scalar dissipation rate $\chi$ is modeled as in [11], including both the resolved and SGS contributions:

$$
\widetilde{\chi}=D_{z}|\nabla \widetilde{Z}|^{2}+D_{t} \frac{C_{\chi}}{2 \Delta^{2}} \widetilde{Z^{\prime \prime 2}}
$$

where $\Delta$ is the filter width and computed as the cuberoot of the cell volume, and $C_{\chi}$ is a model constant set equal to 2 , as in [20, 42]. This modelling approach, with the same constant value, has been recently used in other LES studies employing a similar computational framework [66, 67] albeit in the context of flames under rocket-like conditions. Equivalently, the mixture fraction variance transport equation in $\mathrm{Eq} 2$ can be recast using $\mathrm{Eq} .3$ to yield:

$$
\frac{\partial\left(\bar{\rho} \widetilde{Z^{\prime \prime 2}}\right)}{\partial t}+\frac{\partial\left(\bar{\rho} \widetilde{u_{i}} \widetilde{Z^{\prime \prime 2}}\right)}{\partial x_{i}}=\frac{\partial}{\partial x_{i}}\left[\bar{\rho}\left(D_{z}+D_{t}\right) \frac{\partial \widetilde{Z^{\prime \prime 2}}}{\partial x_{i}}\right]+2 \bar{\rho} D_{t} \frac{\partial \widetilde{Z}}{\partial x_{i}} \frac{\partial \widetilde{Z}}{\partial x_{i}}-\bar{\rho} D_{t} \frac{C_{\chi}}{\Delta^{2}} \widetilde{Z^{\prime \prime 2}},
$$

where the sink term is only function of the sub-grid scale, unresolved, scalar dissipation here modeled resorting to a linear relaxation hypothesis [11]. The effects of subgrid-scale velocity fluctuations are modeled by means of the Smagorinsky model [56].

The numerical setup corresponds to the Sandia/ETH-Zurich experiments of the $\mathrm{CO} / \mathrm{H}_{2} / \mathrm{N}_{2}$ (Flame A) nonpremixed, unconfined, turbulent jet flame [1]. This experimental setup is reproduced at KAUST and installed in the high-pressure combustion test facility operating at elevated pressures. The fuel is injected from a straight circular tube with squared-off ends, with an inner diameter of $4.58 \mathrm{~mm}$ and the outer diameter of $6.34 \mathrm{~mm}$. 


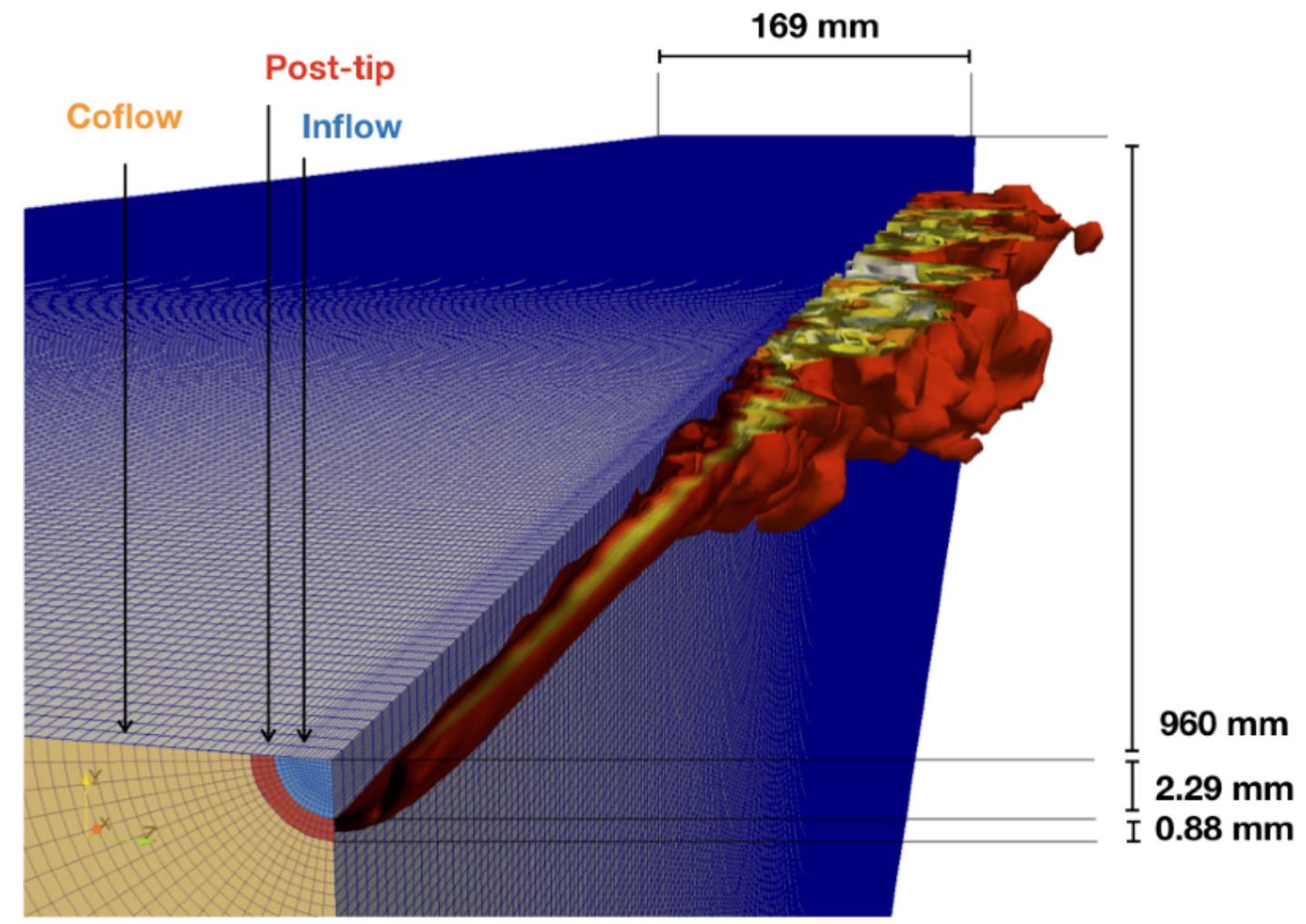

Figure 6: Sector of the numerical domain discretization and temperature iso-contour surfaces.

The geometry and discretization employed for all the numerical computations presented in this work is shown in Fig. 6. The computational domain axial and radial sizes are $960 \mathrm{~mm}(x / D \sim 210)$ and $169 \mathrm{~mm}(x / D \sim 37)$, respectively.

The employed mesh features 26 cells in the jet diameter and 3 cells in the post-tip. Therefore, the initial part of the jet is considered well resolved with at least $\sim 20$ cells in the shear layer region at axial distance $x / D=10$ for the reference case. This led to a total of $\sim 7 \times 10^{6}$ finite volumes, for which a reference grid size of $0.3 \mathrm{~mm}$ was found to be an adequate resolution for all parametric conditions. The level of resolution is comparable to existing simulations of Sandia Flame D ( Re = 22400) by Pitsch et al. [48] and Muller et al. [42], in which 1 and 4 million cells were used, respectively. To our knowledge, 3D LES of the Sandia/ETH-Zurich Flame A ( $R e=16700)$ has not 
been reported in the literature.

The jet inflow mean velocity is modeled as a fully-developed turbulent pipe flow profile, superimposed with prescribed turbulent fluctuations. The lateral boundary conditions are set to freely slip, while an open outflow condition is chosen for the domain outlet boundaries. In all the simulations, the fuel composition is $40 / 30 / 30$ in $\mathrm{CO} / \mathrm{H}_{2} / \mathrm{N}_{2}$ by volume, and is injected at $292 \mathrm{~K}$. The air coflow velocity is $0.75 \mathrm{~m} / \mathrm{s}$, at temperature of $290 \mathrm{~K}$, containing water at mole fraction of $\left.X_{\mathrm{H}_{2} \mathrm{O}}=0.012\right)$.

The validation of the numerical predictions is carried out by comparing with the published Sandia/ETH-Zurich experimental database [1]. The inflow velocity profile employed as inflow condition corresponds to a fully developed turbulent pipe, i.e. $U(r)=U_{\max } r^{1 / n}$, where $r$ is the pipe non-dimensional inner radius $y /(D / 2)$, while $U_{\max }$ is the maximum velocity value. The value of $n$ corresponding to the Reynolds number of interest is 6.6 , while $U_{\max }$ is $105.4 \mathrm{~m} / \mathrm{s}$. In accordance with the experiments, the turbulence intensity is chosen to be $0.1 U_{\max }$ as white noise added to the mean inflow velocity, while the coflow velocity is prescribed at $0.6 \mathrm{~m} / \mathrm{s}$ to match the experimental value. A detailed comparison of the numerical results with experimental data [1] is provided in Appendix A. The numerical setup is shown to successfully reproduce the experimental findings in terms of radial and axial distributions of both mean and root mean square values of velocity, temperature, mixture fraction, as well as both major and minor species concentrations.

\section{Numerical results at elevated pressures}

The LES-SLF numerical setup described in sec. 3.1 has been shown to provide good predictive capabilities in sec. Appendix A. Therefore, together with the a priori assessment of the SLF assumptions reported in sec. 2.3, the same LES-SLF numerical framework can be consistently used for two numerical campaigns, each one consisting of three cases with pressure values of 2, 5, and $10 \mathrm{~atm}$, employing different flamelet look-up tables for each pressure value. In the first campaign, referred to as series A, the inflow velocity is varied with pressure in order to keep the inflow Reynolds number constant. In the second campaign, referred to as series B, the mean inflow velocity is kept constant, with a consequent linear increase of the Reynolds number with pressure. Both 
sets of simulations share the same geometrical configuration of the Sandia/ETH-Zurich Flame A experimental setup.

\subsection{Series A - constant Reynolds number}

A pressure increase leads to a linear increase in density and the dynamic viscosity $\mu$ is independent on pressure. Hence the jet Reynolds number, $R e=\rho U_{j e t} D / \mu$, is kept constant by decreasing the jet velocity $U_{j e t}$ to offset the density increase to retain a constant mass flow rate. The mean inflow velocity condition follows a power-law profile, qualitatively identical to the one employed for the atmospheric test case and described in sec. 3.1, with $U_{\max }$ linearly decreasing with pressure, i.e. $U_{\max }=105.4,52.7,21.08$, and $10.54 \mathrm{~m} / \mathrm{s}$ for pressure values of $1,2,5$, and $10 \mathrm{~atm}$, respectively.
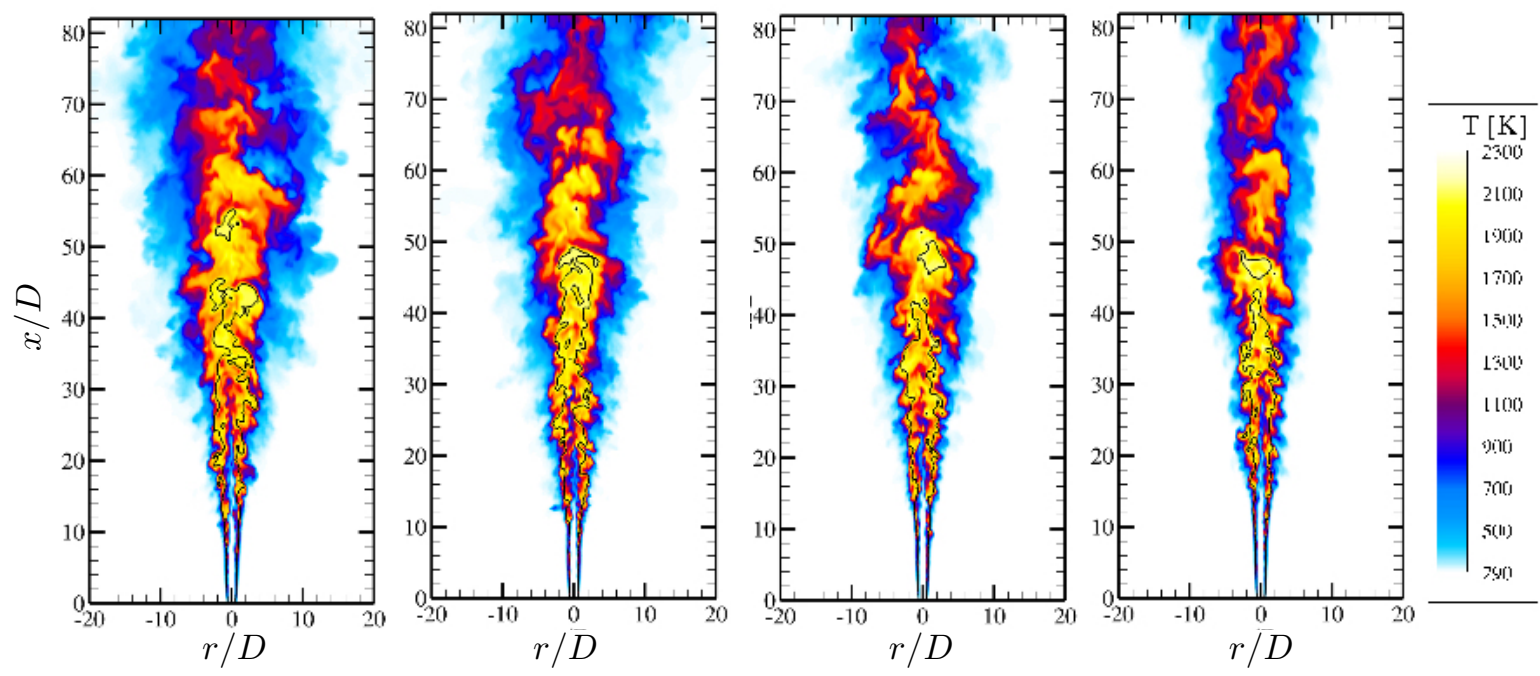

Figure 7: Series A - constant Reynolds number, resolved temperature instantaneous contours, and stoichiometric mixture fraction iso-contour line. From left to right pressure values of 1, 2, 5, and $10 \mathrm{~atm}$.

A first qualitative comparison of the instantaneous resolved temperature fields along with the stoichiometric mixture fraction iso-contour line is shown in Fig. 7. As pressure is increased, we observe a shrinkage of the cyan/blue regions, corresponding to 500 to $1000 \mathrm{~K}$. From the timeaveraged contours shown in Fig. 8, the trend of narrower flame brushes for increasing pressure values is discernible, and a constant flame length is observed. Nonetheless, the maximum value of 

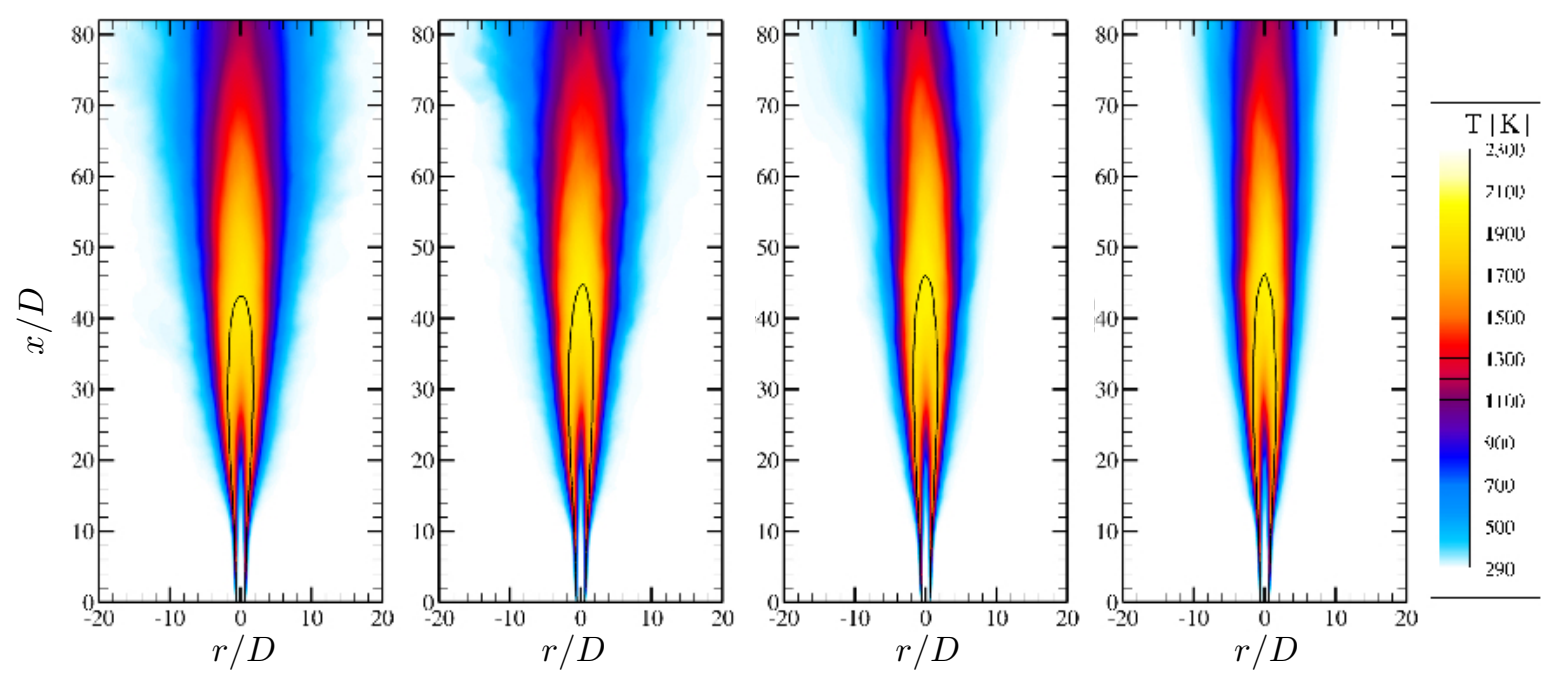

Figure 8: Series A - constant Reynolds number, time-averaged resolved temperature contours, and stoichiometric mean mixture fraction iso-contour line. From left to right pressure values of 1, 2, 5, and $10 \mathrm{~atm}$.

the mean temperature and the mean stoichiochimetric mixture fraction iso-contour line are weakly dependent on pressure.
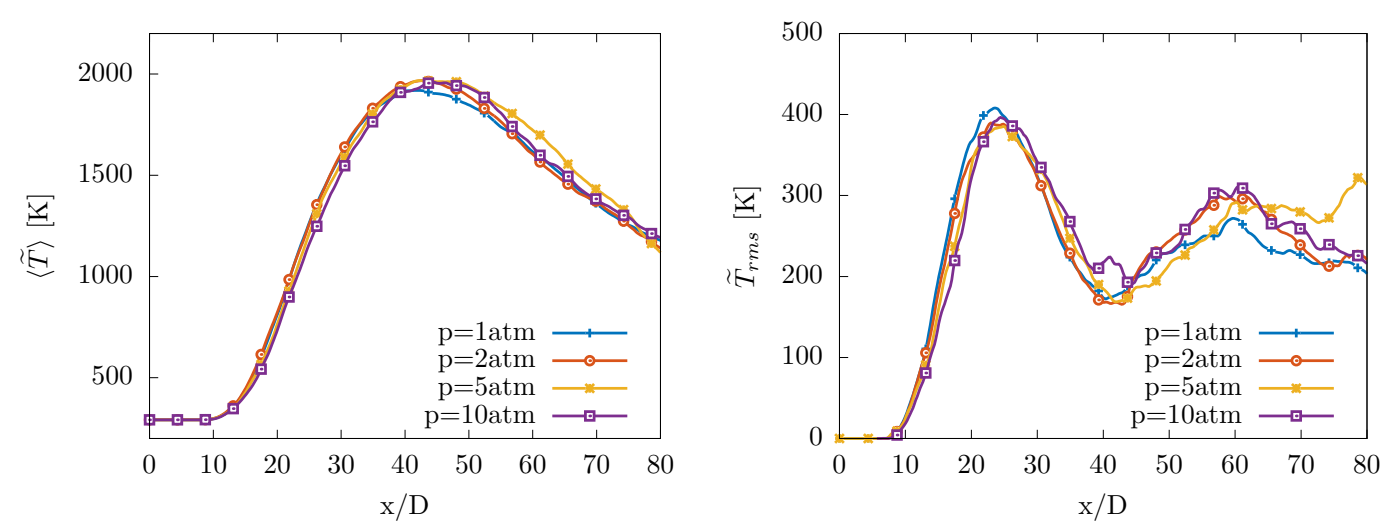

Figure 9: Series A - constant Reynolds number, resolved temperature mean and RMS axial distributions.

The axial distributions of resolved temperature mean and RMS are shown in Fig. 9, where the weak dependence of the maximum flame temperature on pressure is confirmed. As illustrated in Fig. 9 b, temperature RMS exhibits two peaks: the first at $x / D=20$ is associated with small scales 

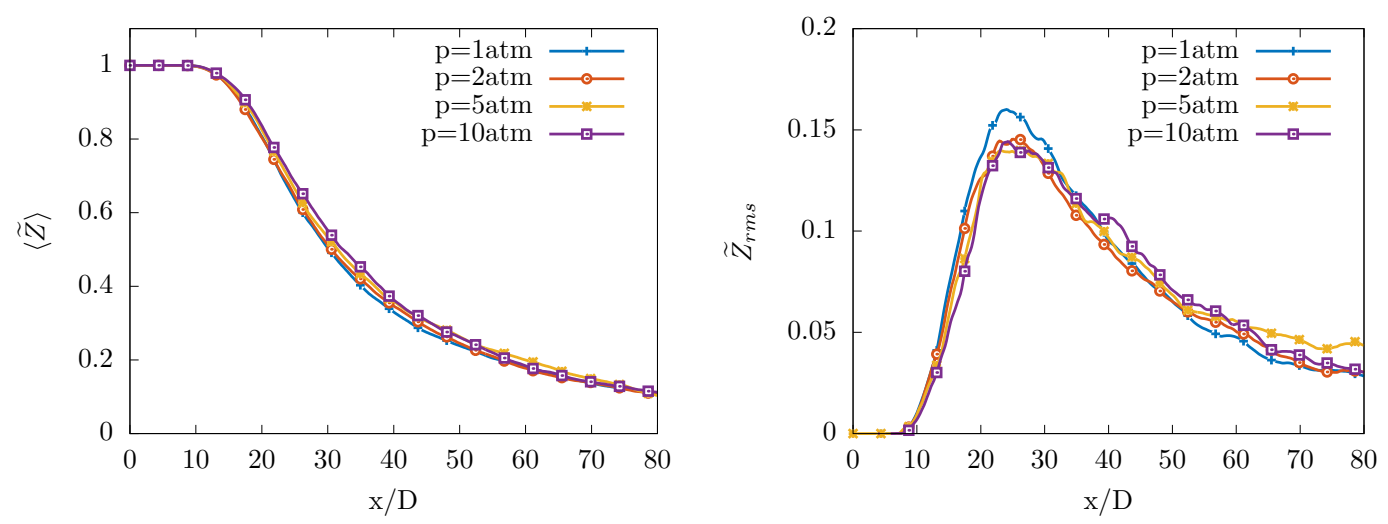

Figure 10: Series A - constant Reynolds number, resolved mixture fraction mean and RMS axial distributions.

oscillations related to turbulent mixing; the second, at $x / D=50$, is due to the alternating detachment of hot products emanating from the jet, possibly due do entrainment effects. At atmospheric conditions, this behaviour is confirmed by the experimental findings as shown in Appendix A, and it is not affected by a pressure increase as long as the mass flow rate and Reynolds number are kept constant. The mean and RMS axial profiles of the mixture fraction are displayed in Fig. 10 , highlighting that the $\langle\widetilde{Z}\rangle$ are weakly dependent on pressure, consistently with the laminar results. In contrast, a mild smearing of the peak of the resolved mixture fraction RMS is observed.

Figure $11 \mathrm{a}$ shows the axial distribution of mean axial velocity normalized by the jet velocity $U_{j e t}$. The axial velocity distributions in the upstream region $(x / D<20)$ appear little affected by a pressure increase, while its value increases gradually with pressure in the downstream region. The RMS of the axial velocity normalized by the jet inflow velocity is shown in Figure $11 \mathrm{~b}$. When pressure is increased, a slight decrease of the normalized velocity RMS is observed, while the RMS peak position is unchanged, the latter confirming that the position of merging point between the mixing layers originating from the injector post-tip does not change among test cases.

Figures $12 \mathrm{a}$ and $12 \mathrm{~b}$ display the radial distributions of the mean mixture fraction and temperature at $x / D=40$, revealing a decrease in the flame brush width is associated with a change in the mixture fraction distribution. This pronounced dependence of temperature distribution on mixture fraction is confirmed in Fig. 12c, where the temperature conditional average on mixture fraction $\langle\widetilde{T} \mid Z\rangle$ at the same axial distance is plotted. Figure $12 \mathrm{c}$ highlights that $\langle\widetilde{T} \mid Z\rangle$ is weakly affected by 


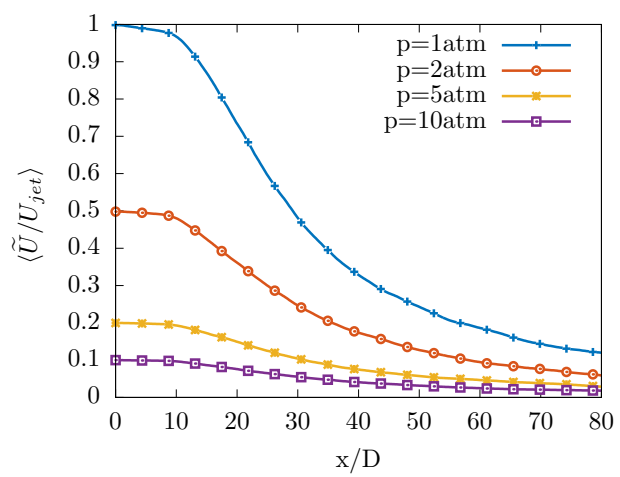

(a)

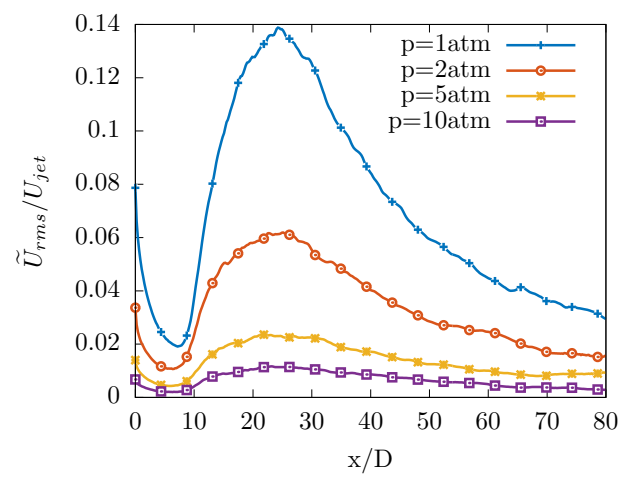

(b)

Figure 11: Series A - constant Reynolds number, resolved numerical results: (a) mean axial velocity normalized by maximum inflow jet velocity; (b) axial velocity RMS normalized by inflow jet velocity.

the pressure change, with the only exception at the stoichiometric region, which exhibits slightly higher temperatures when pressure is increased.

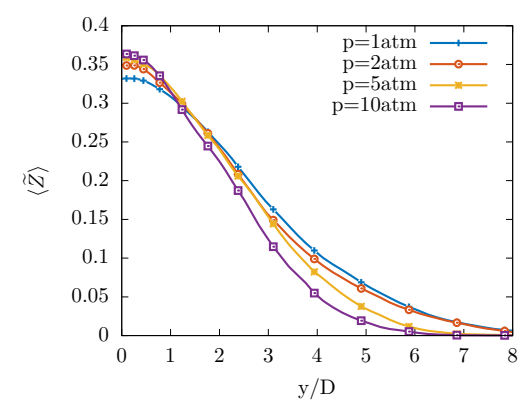

(a)

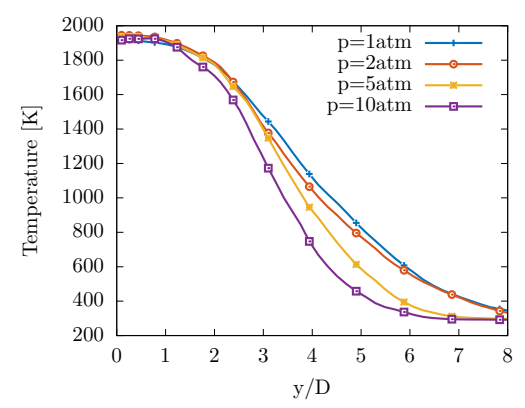

(b)

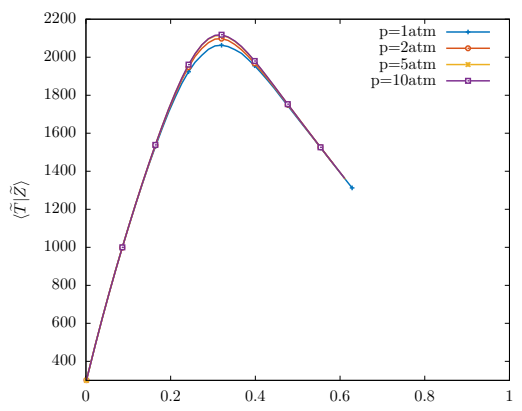

(c)

Figure 12: Series A - constant Reynolds number, resolved numerical results. From left to right: radial distributions of mean mixture fraction and temperature and conditional average of the temperature on mixture fraction at $x / D=40$.

Mean mass fractions of important major and minor species at $x / D=20,40$, and 60 are shown in Fig. 13. The radial distribution of carbon dioxide $\left(\mathrm{CO}_{2}\right)$ and water vapor $\left(\mathrm{H}_{2} \mathrm{O}\right)$ follow the mixture fraction and temperature distributions, and confirm the flame brush narrowing trend with pressure. Moreover, the weak sensitivity of the chemical composition to pressure, observed in sec. 2, is confirmed. The radial distributions of the mean $\mathrm{OH}$ mass fraction are shown, for the same axial distances, in Figures 13g, 13h, and 13i. As expected, also in this turbulent scenario, 
the pressure increase pushes composition toward chemical equilibrium, causing a decrease in the radical species such as $\mathrm{OH}$, consistent with the findings in sec. 2 for the laminar diffusive flames.

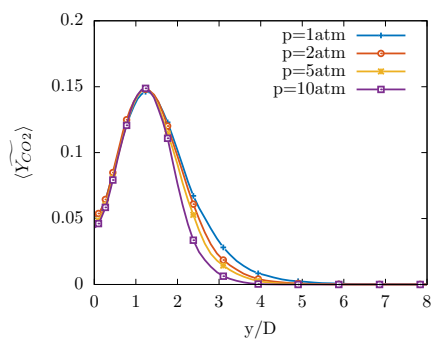

(a) $x / D=20$

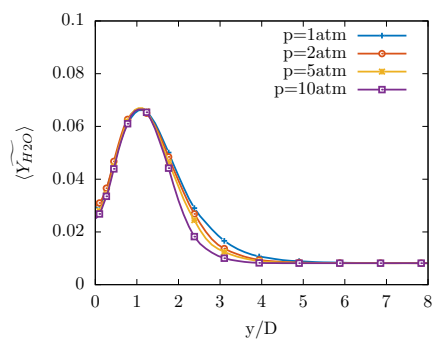

(d) $x / D=20$

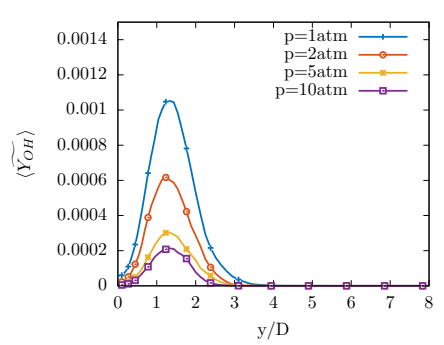

(g) $x / D=20$

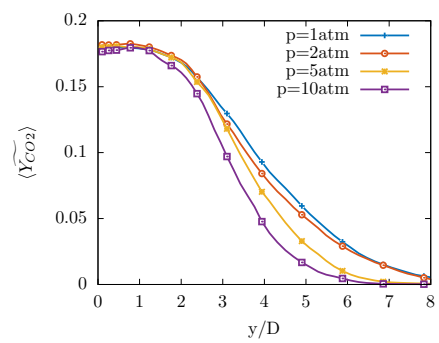

(b) $x / D=40$

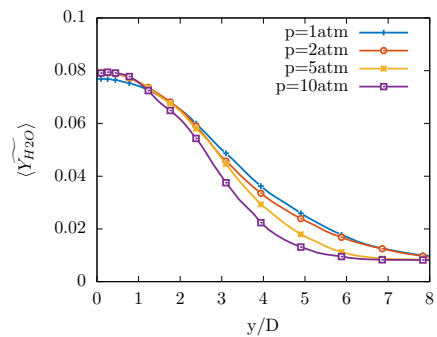

(e) $x / D=40$

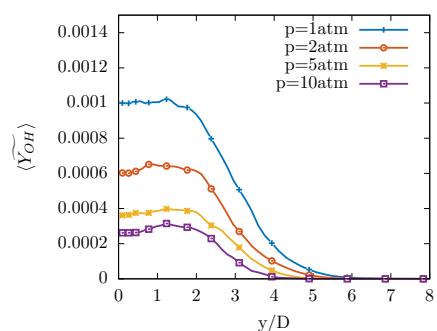

(h) $\mathrm{x} / \mathrm{D}=40$

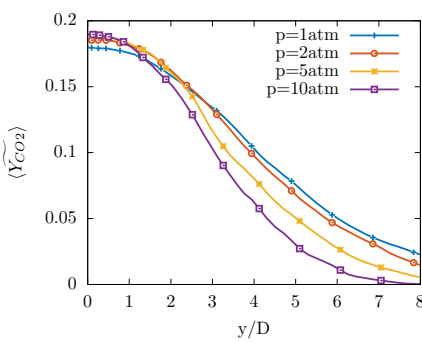

(c) $x / D=60$

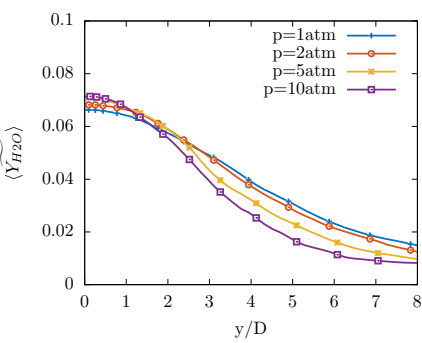

(f) $x / D=60$

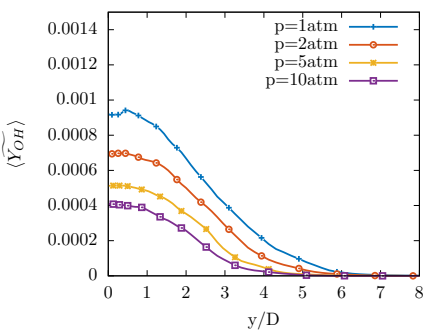

(i) $\mathrm{x} / \mathrm{D}=60$

Figure 13: Series A - constant Reynolds number, resolved numerical results. Radial profiles of $\mathrm{CO}_{2}, \mathrm{H}_{2} \mathrm{O}$, and $\mathrm{OH}$ mass fraction mean values at normalized distances from the inlet of $x / D=20,40$ and 60 .

\subsection{Series $B$ - constant mean inflow velocity}

The same numerical setup was employed for three additional simulations for pressure values of 2,5 , and $10 \mathrm{~atm}$, in which the same jet mean velocity of flame A is selected. In this series B, since the mean velocity is kept constant and the dynamic viscosity is independent on pressure, the pressure increase implies a linear Reynolds number increase through density. Hence, the fullydeveloped turbulent pipe flow velocity profile described in sec. Appendix A is modified according 
to the increased Reynolds number with values of $n=6.6,6.7,6.9$ and 7.1, for $R e=16700,33400$, 83500 , and 167000 , corresponding to $p=1,2,5,10 \mathrm{~atm}$, respectively. To ensure the same inflow mean velocity is obtained, the maximum velocity is varied to account for the variation in the inflow velocity profiles with pressure, with $U_{\max }=105.4,104.684,104.08$, and $103.5 \mathrm{~m} / \mathrm{s}$ in order of increasing pressure.

The increase in the jet Reynolds number may lead to a poor resolution of the highest Re case. To assess the adequate resolution for the highest Re case, we refer to the cold flow experimental tests on the same configuration at increasing pressures [6]. It was reported that as pressure increases while the inflow velocity is fixed, the integral length scale of the turbulent flow field remains approximately constant. On the other hand, the increase in Re leads to a decrease in the Kolmogorov length scales. Following the same reasoning [6], the turbulence length scales of interest are estimated to be $\lambda \sim 0.157 \mathrm{~mm}$ and $\eta \sim 0.003 \mathrm{~mm}$. Recalling that our LES filter is about $\sim 0.3 \mathrm{~mm}$, it is concluded that even the highest Re case ( $p=10$ atm of series B ) is adequately resolved.
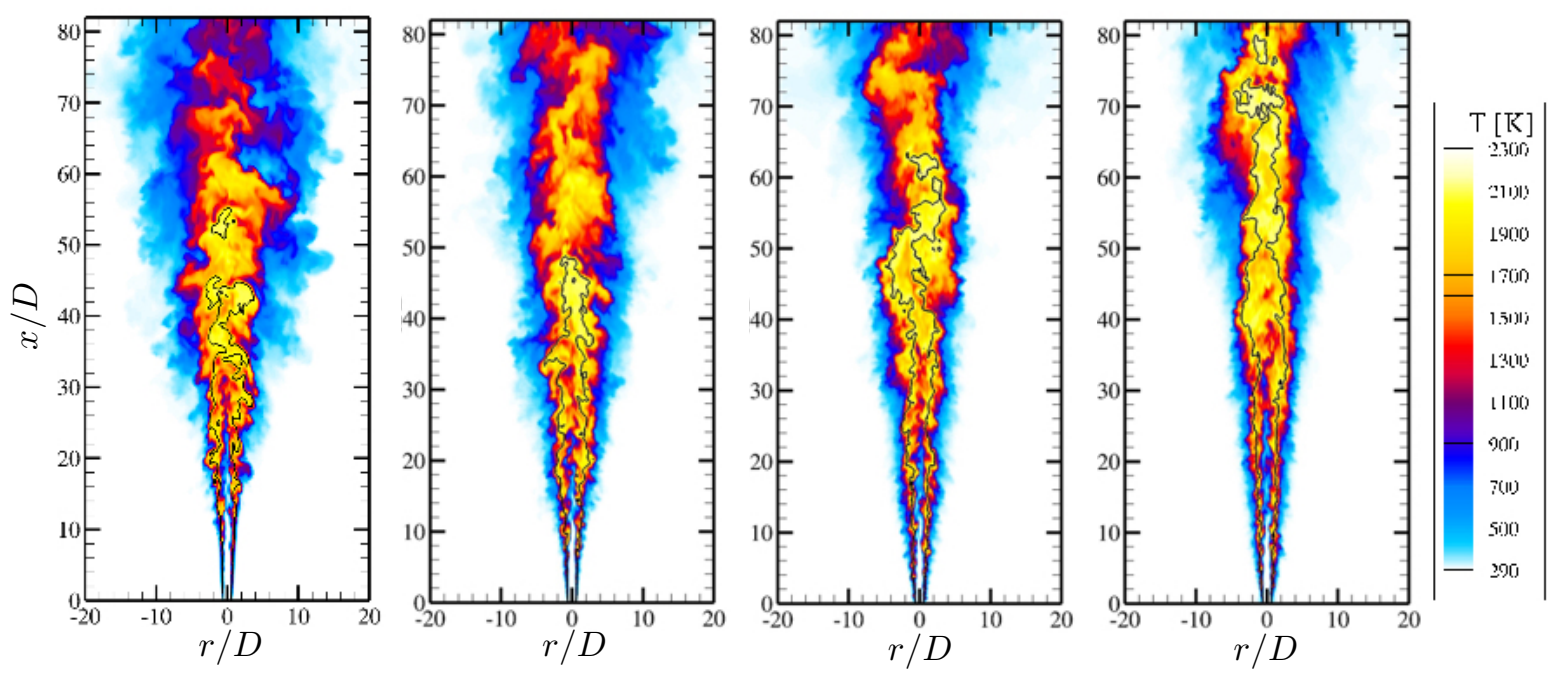

Figure 14: Series B - constant mean inflow velocity, resolved temperature instantaneous contours, and stoichiometric mixture fraction iso-contour line. From left to right pressure values of 1, 2, 5, and $10 \mathrm{~atm}$.

Representative instantaneous temperature fields are displayed in Fig. 14. Similarly to series 

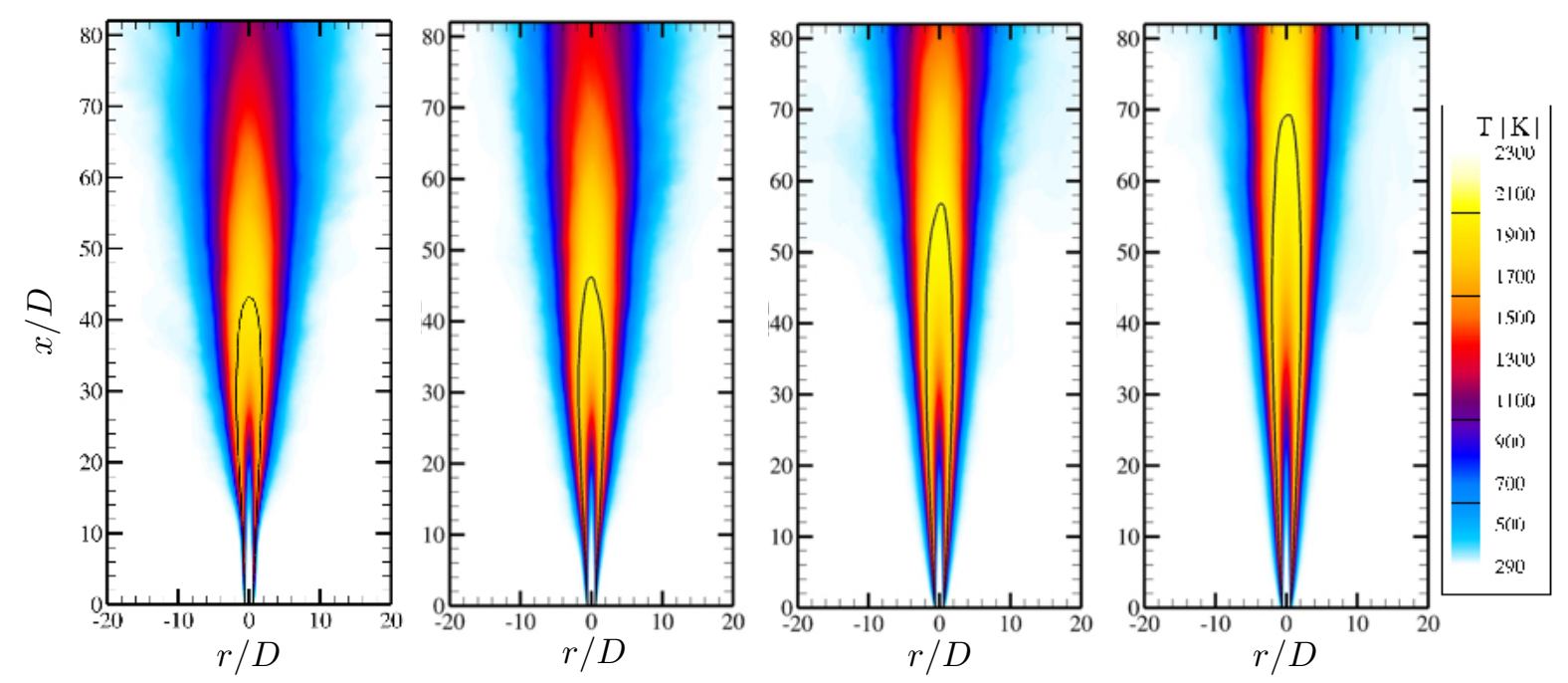

Figure 15: Series B - constant mean inflow velocity, time-averaged resolved temperature contours, and stoichiometric mean mixture fraction iso-contour line. From left to right pressure values of 1, 2, 5, and $10 \mathrm{~atm}$.
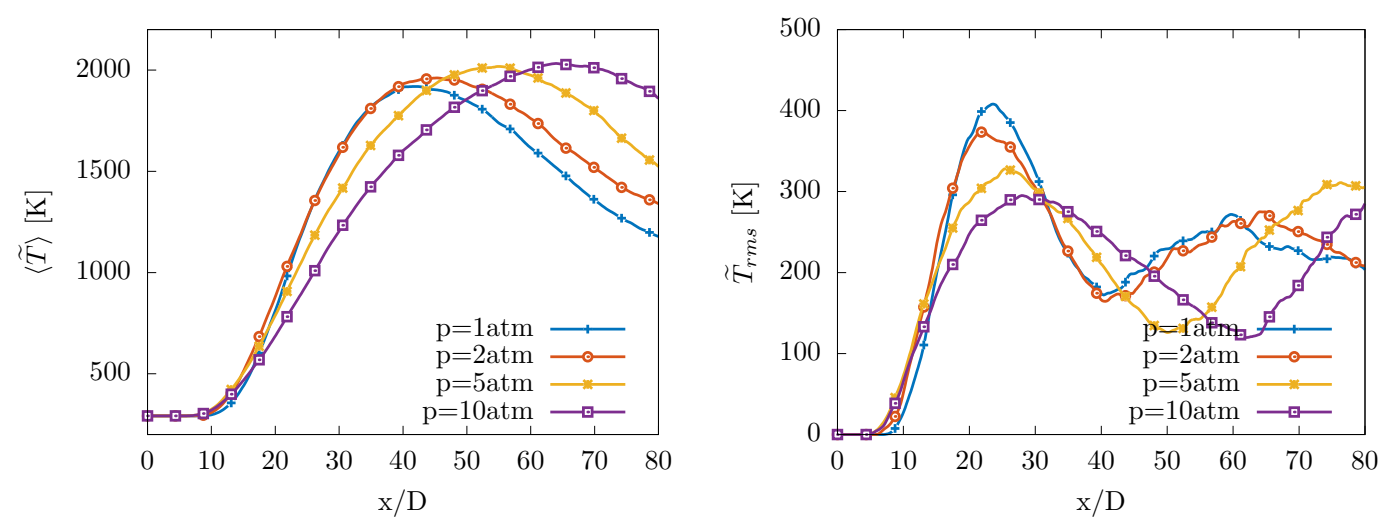

Figure 16: Series B - constant mean inflow velocity, resolved numerical results: temperature mean and RMS axial distribution.

A, the extent of the cyan/blue regions, corresponding to temperatures ranging between $500 \mathrm{~K}$ and $1000 \mathrm{~K}$, diminishes with pressure. Moreover the light yellow regions, corresponding to temperatures higher than $2000 \mathrm{~K}$, occupy a larger part of the domain. In contrast to series A, the flame length increases with pressure and the high temperature region is less fragmented and is broadened in the axial direction. This increase in the flame length is clearly visible by the mean stoichiometric 

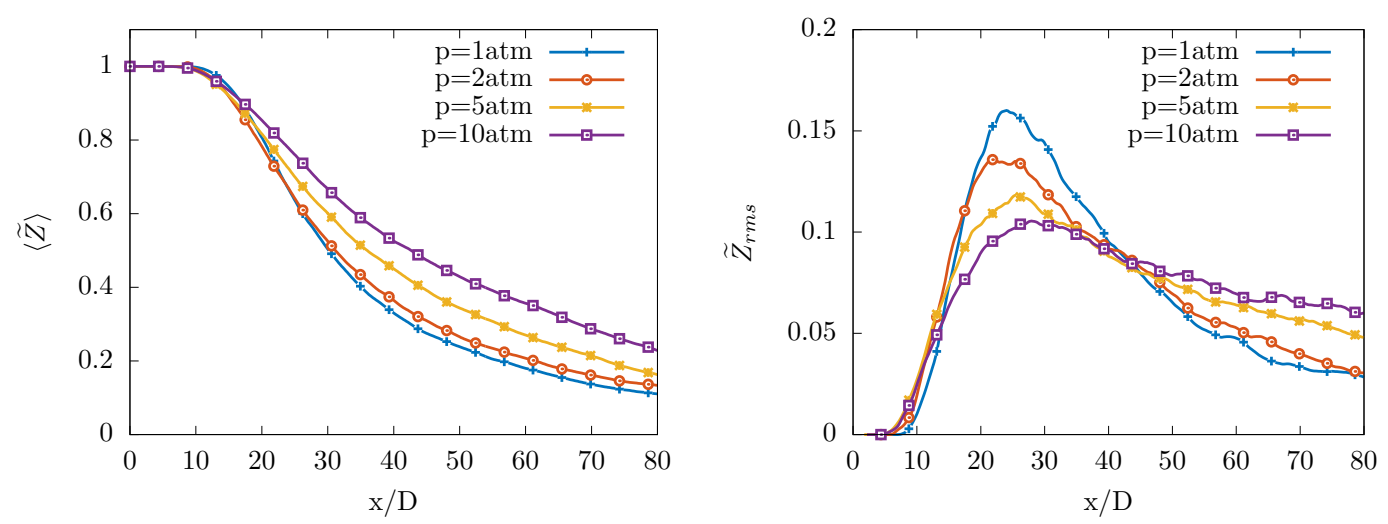

Figure 17: Series B - constant mean inflow velocity, numerical results: mixture fraction mean and RMS axial distribution

mixture fraction iso-contours in Fig. [15, where the mean temperature contours are also displayed.

The axial distributions of mean and RMS values of the resolved temperature are shown in Fig. 16, where the effects of pressure, i.e. of the increased mass flow rate, on the flame length are clearly observable, with the maximum temperature being shifted downstream. The temperature RMS distribution shows two peaks, qualitatively similar to the atmospheric case, but are shifted downstream as pressure increases. While the magnitude of the first peak around $x / D \sim 20-30$ is reduced by the pressure increase, the second one beyond $x / D \sim 60$ appears to be more pronounced. The mean and RMS of mixture fraction are shown in Fig.17 where the increase of mixture fraction values in the axial regions downstream $x / D=20$ and the diminishing of the RMS peak are consistent with temperature mean and RMS distributions.

The axial distribution of mean and RMS values of the axial velocity, and the RMS of the radial velocity are shown in Fig. 18, where an increase in the mass flow rate results in a longer flame brush. In particular, Figures $18 \mathrm{~b}$ reveals a decrease in the RMS of axial and radial velocity fluctuations, whose peak indicates the merging point of the shear layers originating at the injector post-tip.

In Figures 19a and 19b, the radial distributions of the mean mixture fraction and temperature at $x / D=40$ are plotted, indicating that the flame brush narrowing is associated with changes in the mixture fraction distribution, as confirmed by the temperature conditional average on mixture 


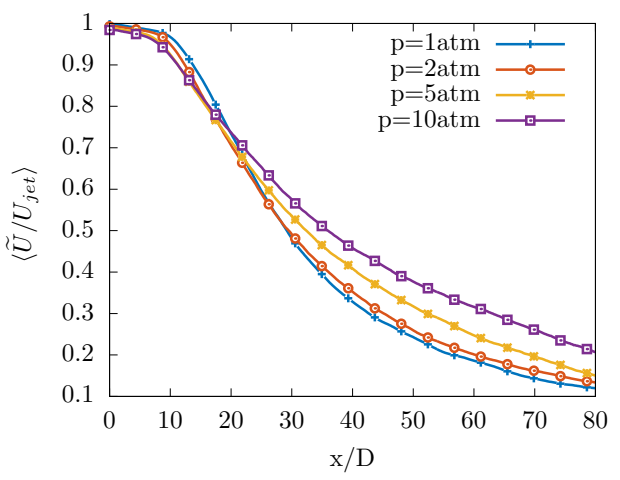

(a)

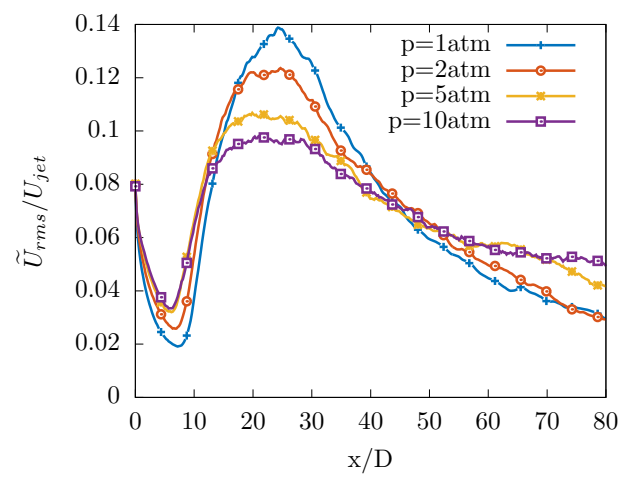

(b)

Figure 18: Series B - constant inflow velocity, resolved numerical results: (a) axial distributions of: mean axial velocity normalized by inflow jet velocity; (b) axial velocity RMS normalized by inflow jet velocity.

fraction $\langle\widetilde{T} \mid \tilde{Z}\rangle$ at the same axial distance reported in Fig. $19 \mathrm{c}$, where the distribution of the temperature conditional average as function of the mixture fraction is unaffected by the pressure change. Moreover, the radial distribution of the mean temperature in Fig. 19b, along with the mean temperature contours in Fig. 15, highlight that the merging point of the mixing layer originated at the injector post-tip is slightly shifted downstream when pressure is increased.

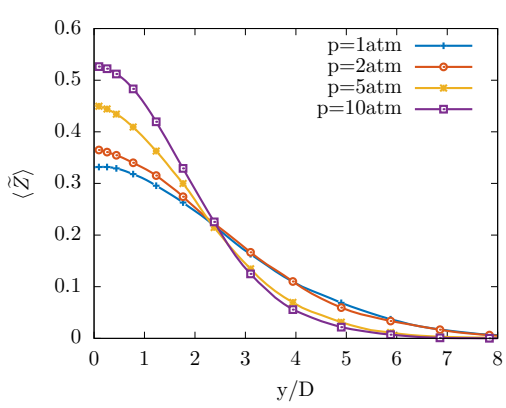

(a)

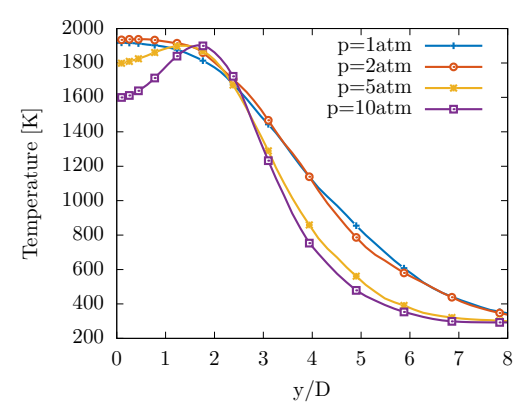

(b)

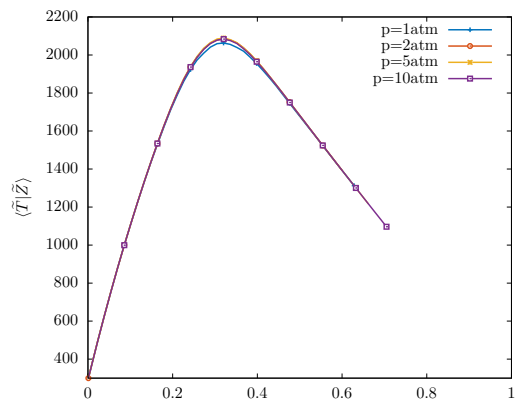

(c)

Figure 19: Series B - constant inflow velocity, resolved numerical results. From left to right: radial distributions of mean mixture fraction and temperature and conditional average of the temperature on mixture fraction at $x / D=40$.

Finally, the radial distribution of important major/minor species mass fractions at $x / D=20$, 40, and 60 are shown in Fig. 20. The mean profiles of the major combustion products mass fractions, namely $\mathrm{H}_{2} \mathrm{O}$ and $\mathrm{CO}_{2}$, are consistent with the mixture fraction and temperature distributions, 
and confirm that the flame brush becomes narrower and longer when pressure is increased. Also in this series $\mathrm{B}$, the overall presence of the $\mathrm{OH}$ radical is reduced as the pressure increases. In fact, the concentration of $\mathrm{OH}$ is reduced by both fluid dynamic and chemical effects; the former induces a narrower flame region in the physical space, while the latter shifts the reacting mixture toward chemical equilibrium in the composition space.

\section{Comparison with experiments}

Boyette et al. [6] experimentally investigated the effects of pressure and Reynolds number on the structure of turbulent non-premixed flames. Analogous to the present work, the baseline atmospheric pressure flame is the Sandia/ETH Flame A from the Turbulent Nonpremixed Flames (TNF) Workshop [1]. In the experimental campaign, Syngas (CO/H2/N2) flames with constant composition were examined at pressures up to 12 atm and Reynolds numbers up to 66,800. Unlike the numerical study presented here, however, the experimental work focused on the flame structure in the region where $5 \leq x / D \leq 8.2$, investigated by means of Low-speed OH-PLIF. The main outcomes of the experimental work were: i) local extinction was prevented with increasing pressure at constant Reynolds number; ii) a decrease in the $\mathrm{OH}$ layer thickness was found with increasing pressure; and iii) the flame position was insensitive to pressure.

To provide a comparison between the numerical and the experimental findings, the above outcomes are investigated within the numerical results and discussed in the following.

The local extinction is expected to occur when the local strain rate, or scalar dissipation rate $\chi$, is higher than a critical value, referred to as quenching scalar dissipation rate, $\chi_{q u}$. In the laminar one-dimensional study in $\operatorname{Sec} 2$, it was demonstrated that a pressure increase leads to an increase in $\chi_{q u}$, as shown in Fig. 4. Figure 22 illustrates the mean of the scalar dissipation rate, conditional on the mixture fraction, for all pressures in both series $\mathrm{A}$ and $\mathrm{B}$, showing a monotonic decrease in the scalar dissipation rate with pressure. Moreover, the RMS of the scalar dissipation rate is also found to decrease with pressure, in the entire domain and in both series A and B, as reported in Fig.23. Combined with the fact that the chemical reactivity increases with pressure, the net result is that an increase in pressure leads to a flame more resistant to extinction. Consequently, local 


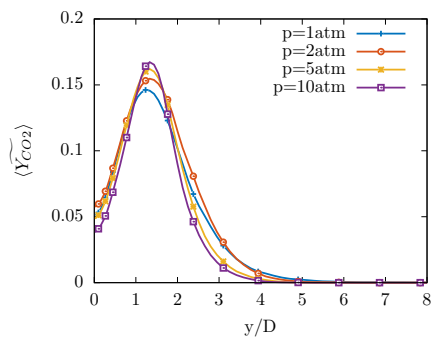

(a) $x / D=20$

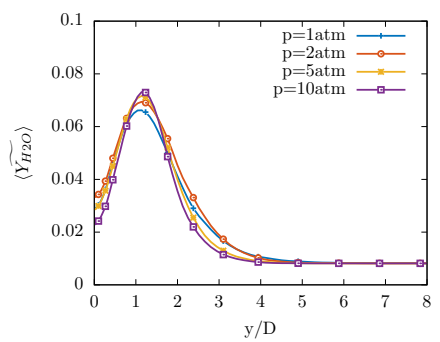

(d) $x / D=20$

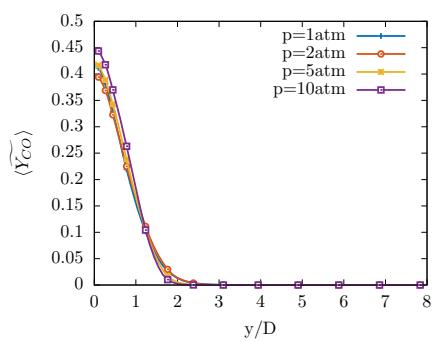

(g) $x / D=20$

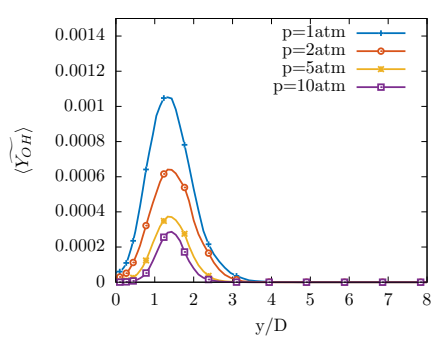

(j) $x / D=20$

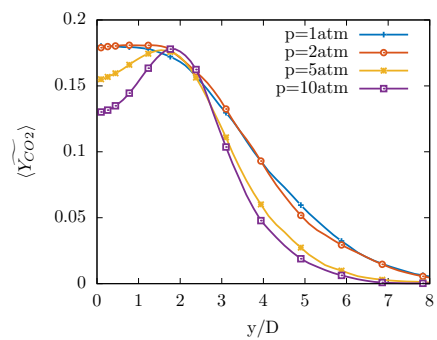

(b) $x / D=40$

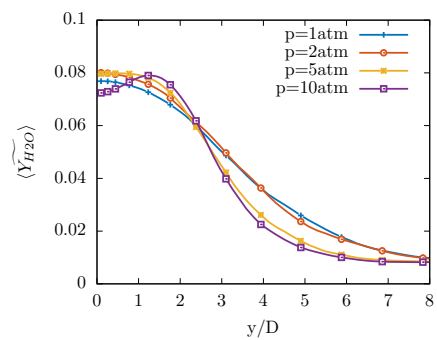

(e) $x / D=40$

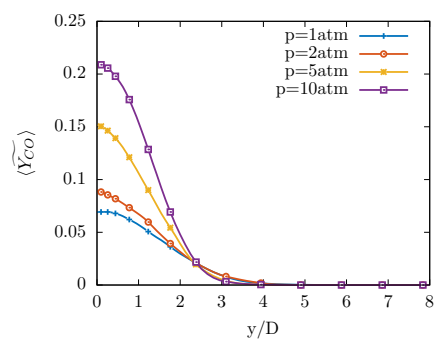

(h) $x / D=40$

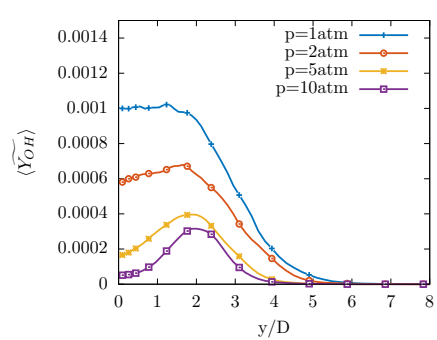

(k) $x / D=40$

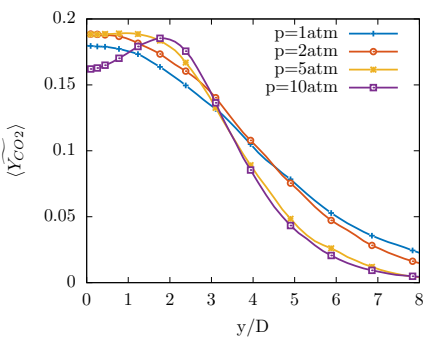

(c) $x / D=60$

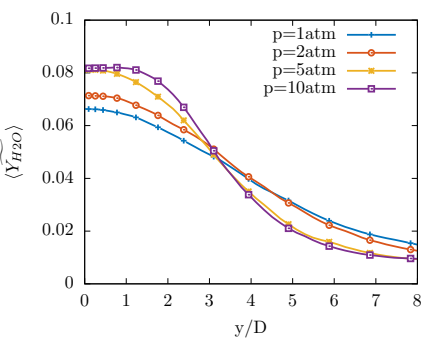

(f) $x / D=60$

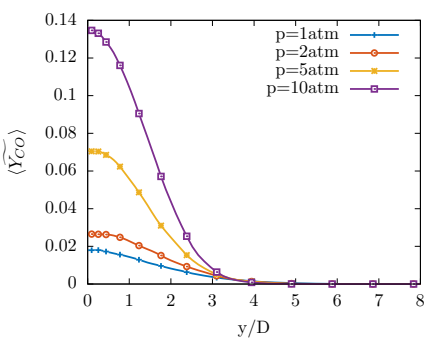

(i) $x / D=60$

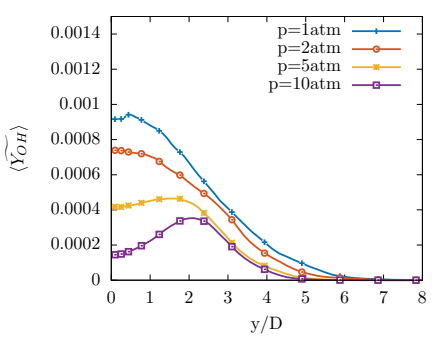

(1) $x / D=60$

Figure 20: Series B - constant inflow velocity, resolved numerical results. Radial profiles of $\mathrm{CO}_{2}, \mathrm{H}_{2} \mathrm{O}, \mathrm{CO}$, and $\mathrm{H}_{2}$ mass fraction mean values at normalized distances from the injector of $x / D=20,40$ and 60 .

extinction not observed in all cases under study, consistent with the observations by Barlow et al. [1] in their experimental work at atmospheric conditions. 


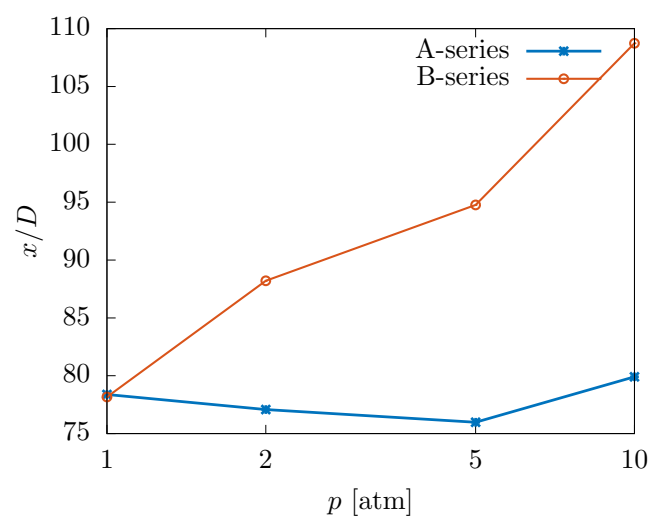

(a) Axial length

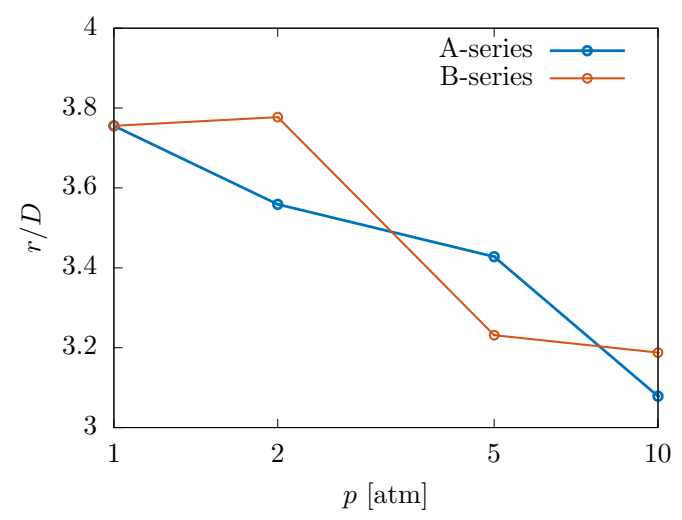

(b) Radial width at $\mathrm{x} / \mathrm{D}=40$

Figure 21: Axial length and radial width of the turbulent flame investigated, both the characteristic flame dimensions have been calculated using the $\langle\widetilde{T}\rangle=1200 \mathrm{~K}$ iso-contour line.

Fig. 24a shows the flame thickness computed on the basis of the $\mathrm{OH}$ layer thickness definition of Canny et al. [16], compared with the experimental values for the constant Reynolds cases. The LES results inherently give spatially filtered values, while the experimental measurements are also subjected to the limitations in the resolution. Within the uncertainties, the average $\mathrm{OH}$ layer thickness is quantitatively in good agreement with the experimental results. Furthermore, the $\mathrm{OH}$ layer thickness decreases monotonically with pressure up to $5 \mathrm{~atm}$, consistent with the experimental trend, and then levels off from 5 to $10 \mathrm{~atm}$. The latter behaviour is possibly attributed to the mesh resolution of the order of $300 \mu \mathrm{m}$ approaching the limit of resolving the fine structures. Nevertheless, the $\mathrm{OH}$ layer attenuation with pressure is also consistent with the results from the laminar counterflow computation.

Finally, the flame position, here defined as the locus of points of maximum $\mathrm{OH}$ concentration, is shown in Fig $24 \mathrm{~b}$ in terms of mean value and standard deviation. It is seen that the flame position is essentially not sensitive to pressure, staying at $y / D=0.7-0.8$, again consistent with the experimental findings.

\section{Discussion}

Given the limited amount of available validation data, and considering the challenges in the 


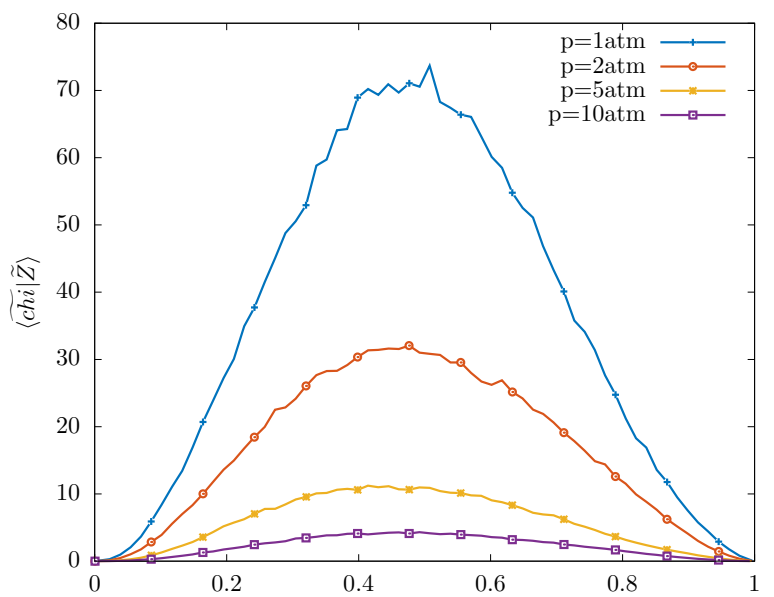

(a)

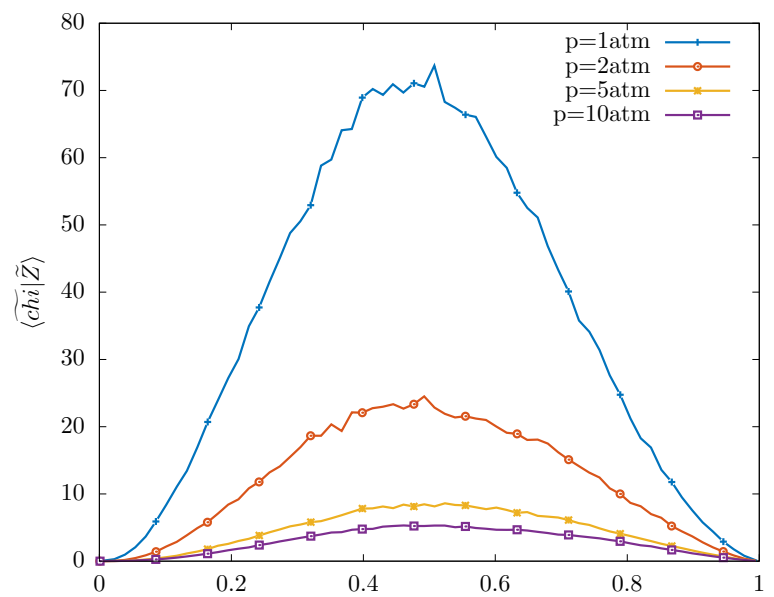

(b)

Figure 22: Series A left and series B right - conditional average on the mixture fraction $Z$ of the scalar dissipation rate at $x / D=10$.

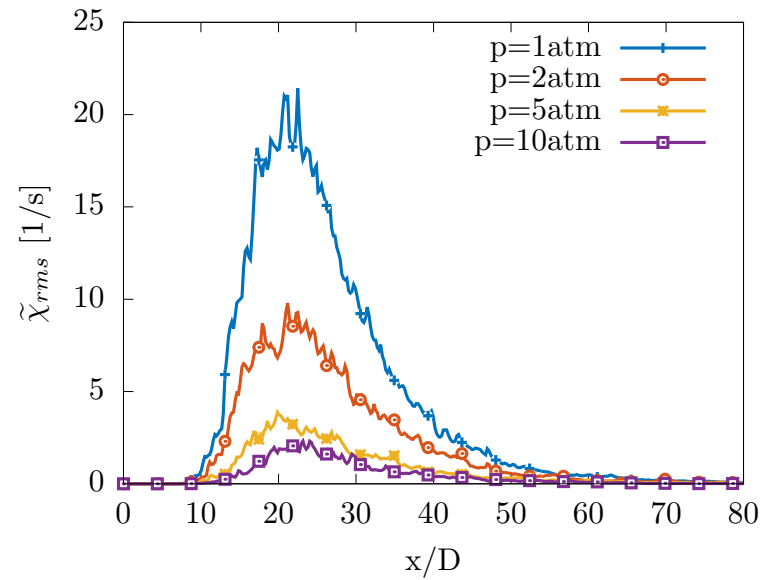

(a)

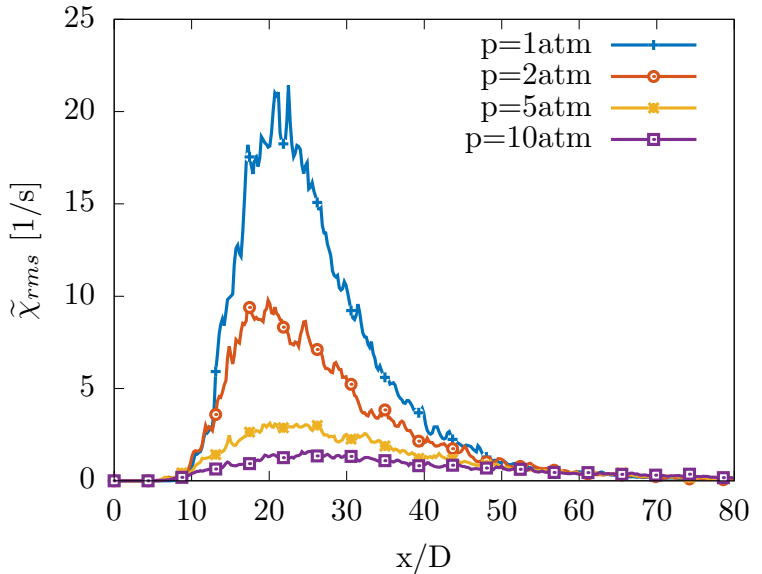

(b)

Figure 23: Series A left and series B right - the distribution of the standard deviation of the scalar dissipation rate on the axial direction for all pressures.

quantitative diagnostics at such conditions and the time required to acquire the next set of detailed data, the numerical results represent a logical step to guide future directions of the experimental work. For this purpose, we present and discuss global and local features of the numerically 


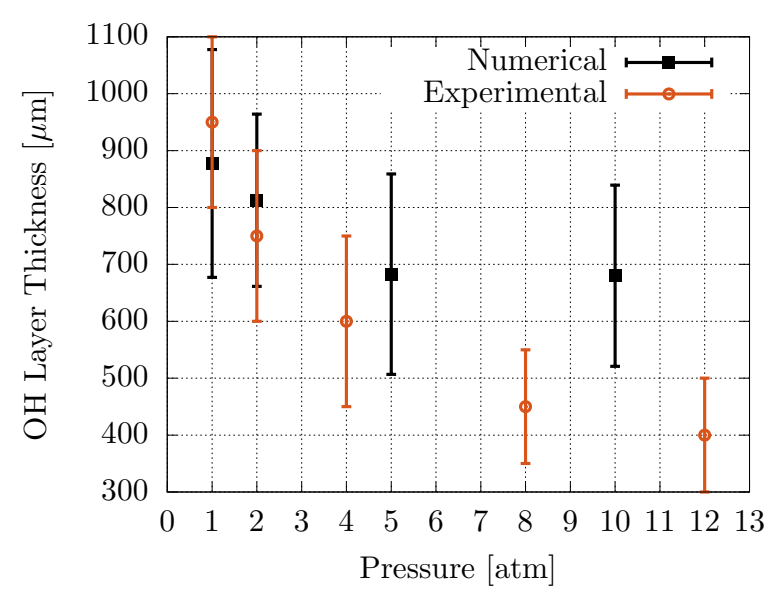

(a)

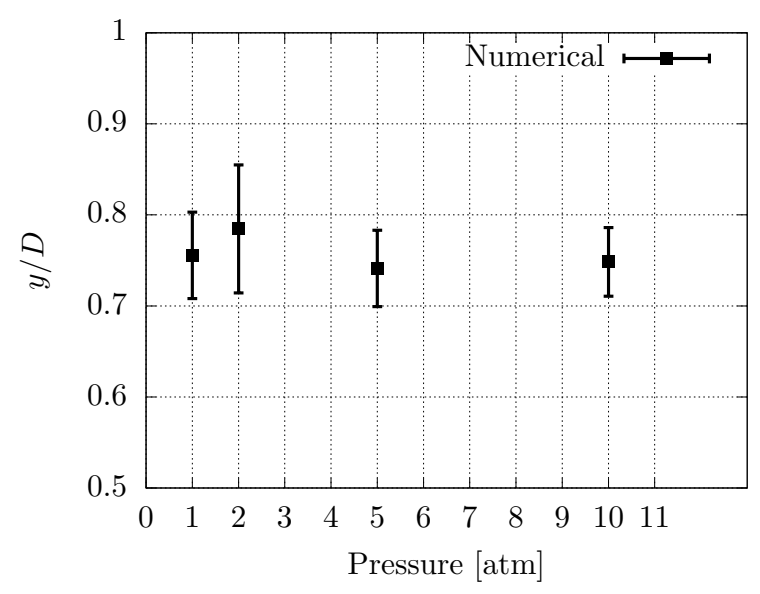

(b)

Figure 24: Series A. Left: $\mathrm{OH}$ layer thickness as a function of pressure at $x / D=6$. Right: flame position as a function of pressure.

computed turbulent flames, and their sensitivity to pressure.

\subsection{Pressure effects on scalar dissipation rate}

To support the a priori assessment of SLF-LES applicability discussed in sec. 2.3, the sensitivity of the mixing time scales, i.e the scalar dissipation rate, to pressure is examined. Scalar dissipation rate was found to decrease with pressure in both campaigns in the entire domain. As an example, the conditional average on mixture fraction of the scalar dissipation rate at $x / D=10$ is shown in Figs. 22a and 22b. To leave no doubts on a possible local rise in the scalar dissipation rate due to turbulent fluctuations, the distribution of the standard deviation of the scalar dissipation rate on the axial direction for all pressures is reported in Fig. 23, which shows that the values are overall much lower than the mean, and further diminishing with pressure in both numerical campaigns.

\subsection{Global flame features}

As expected from laminar calculations, in both campaigns, pressure plays an important role in pushing the composition towards chemical equilibrium, causing a diminution of the radical species such as $\mathrm{OH}$. 
In series A, a narrower flame brush with increasing pressure is observed, while the jet flame length results independent on pressure. Furthermore, the maximum value of the mean temperature is found to to be weakly dependent on pressure. The hot-pocket shedding dynamics, observed also at atmospheric conditions, is also present at higher pressure as indicated by the two peaks

in the $\widetilde{T}_{r m s}$ axial distribution. The sensitivity of the RMS peak values on pressure appears to be negligible. In series B, a narrower jet flame with increasing pressure has been observed, similarly to series A. On the other hand, the increased mass flow rate results in a higher flame length. A summary of the main geometrical flame differences is reported in Fig. 21. The iso-contour line corresponding to $T=1200 \mathrm{~K}$ is chosen as reference to define the spatial extension of the flame. In Fig. 21a the flame length is reported as a function of pressure, confirming that the flame length increase is significant for series B moving from $x /\left.D\right|_{\widetilde{T}=1200}=77$ at atmospheric conditions to $x /\left.D\right|_{\widetilde{T}=1200}=108$ at $p=10 \mathrm{~atm}$, while the flame length variation is much less pronounced in series A. The flame width at $x / D=40$ is reported in Fig. $21 \mathrm{~b}$ resulting in a mild width decrease with pressure both in series A and B.

\subsection{Self-similarity correlations}

This section shows comparisons with the correlation studies proposed by Richards and Pitts [53] which experimentally investigated the global density effects on self-similarity characteristics of turbulent jets. Self-similarity is not recovered in flows in which the density varies appreciably across the flow; that is, self-preserving solutions do not exist for the governing equations. Nonetheless, Richards and Pitts [53] proved that axisymmetric jets with an initial density different than that of the ambient are characterized by density gradients decreasing rapidly with the distance from the inflow orifice, because of the entrainment process, and the flow approaches a state in which the density ratio between the local jet fluid and the ambient approaches unity. On one hand, a self-similar behaviour is not expected in the case of nonpremixed diffusive flames, for which a strong heat release affects the shear layer where stoichiometric conditions are reached; on the other hand, a significant insights can be drawn by the comparison of the non reacting and the reacting jet behaviour, in terms of axial variation of both mixture fraction and velocity. We remark that all the computations are designed to mimic the experimental facility, which is bounded 
to maintain the prescribed pressure value. This is an additional cause of discrepancy with the expected self-similar behavior, only possible for unbounded jets.

In open-bounded cold flows, the jet growth is linear in the axial direction, and the center-line decay of the mass fraction should result to be inversely proportional to the axial distance, following the relation:

$$
\langle Z(x, 0)\rangle^{-1}=\frac{K_{c}\left(x-x_{0, Z}\right)}{R} .
$$

where $\langle Z(x, 0)\rangle$ is the average mixture fraction as a function of the axial coordinate $x$ at the centerline, $K_{c}$ and $x_{0, Z}$ are the rate of centerline decay and the virtual origin of the jet and are usually found experimentally [51]. First, the axial behaviour of $\langle Z\rangle^{-1}$ along the centerline is reported as a function of the normalized axial coordinate $x / R$ in Fig $25 \mathrm{a}$ for series-A and Fig $25 \mathrm{~b}$ for series-B. As a reference, both plots are reported the atmospheric Sandia/ETH-Zurich Flame A measurements [1] and the reference cold jet behaviour as per Eq.5, with $K_{c}=0.1$ and $x_{0, Z} / R=8$. Fig 25 clearly shows that the behaviour of $\langle Z\rangle^{-1}(x)$ at atmospheric pressure agrees with the reference atmospheric experimental measurements, and it is not linear in the region going from the jet orifice to approximately $x / R=100$, after which the trend seems to recover a linear nature. This is consistent with the expectation of having self-similar behaviour at a distance from the inflow orifice sufficiently large to imply low radial density gradients due to turbulent entrainment of ambient air. As expected, the effects of pressure on this behaviour seem negligible when the Reynolds number is kept constant, as a reference significant pressure-induced differences in the evolution of iso-Re jets have been recently observed [28] under peculiar thermodynamic conditions [29, 32]. Conversely, longer jets are observed when Reynolds number is increased with pressure is confirmed by the $\langle Z(x, 0)\rangle^{-1}$ in Fig $25 b$, where a pressure increase results in a decrease of the slope of the curves, and the linear trend is recovered further downstream.

The half-widths of mixture fraction $r_{\frac{1}{2}, Z}$ and axial velocity $r_{\frac{1}{2}, U}$ are defined as the radial locations at which mixture fraction or axial velocity, respectively, attain half the value reached at the center-line position. The nondimensional mixture fraction half-width, $r_{\frac{1}{2}, Z} / R$, for both series $\mathrm{A}$ and $\mathrm{B}$ is reported in Figures 26a and 26b, respectively, as a function of the non-dimensional axial distance $x / R$. As a reference, the corresponding experimental measurements of Sandia/ETH-Zurich 


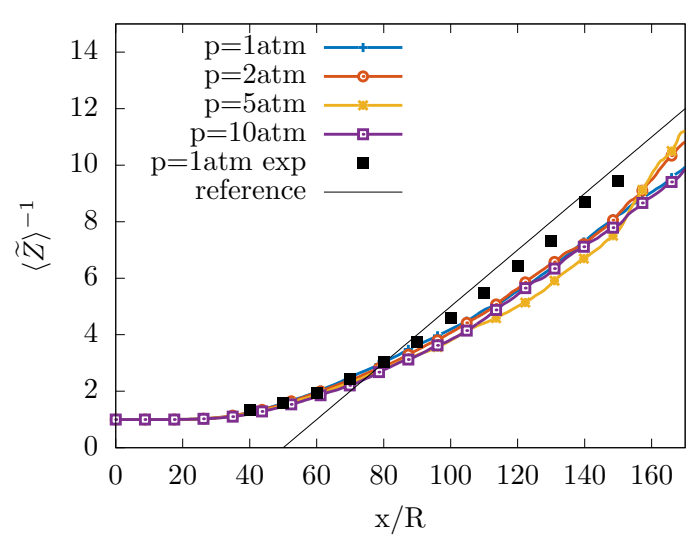

(a) series-A constant Reynolds number

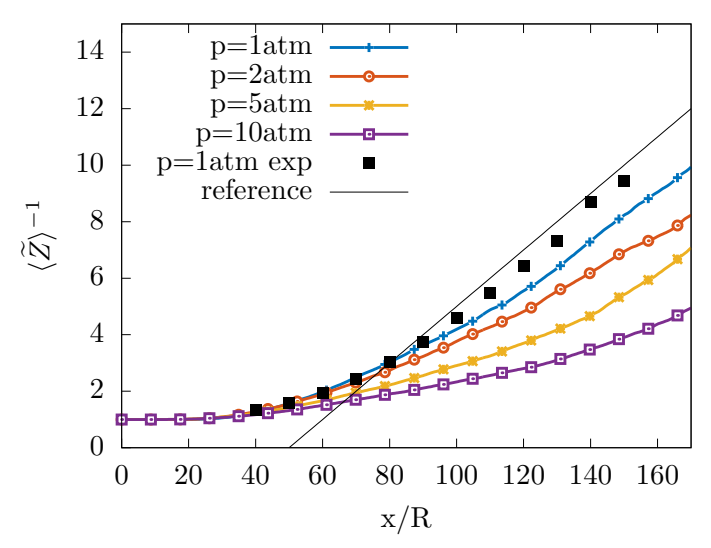

(b) series-B constant inflow velocity

Figure 25: Reciprocal of the mixture fraction axial distribution along the centerline as a function of the nondimensional axial coordinate $x / R$ for: series A and B; atmospheric reference Sandia/ETH-Zurich Flame A experiment[1]; typical cold-jet linear behaviour $\langle Z(x, 0)\rangle^{-1}=\frac{K_{c}\left(x-x_{0, Z}\right)}{R}\left(K_{c}=0.1, x_{0, Z} / R=8\right)$.

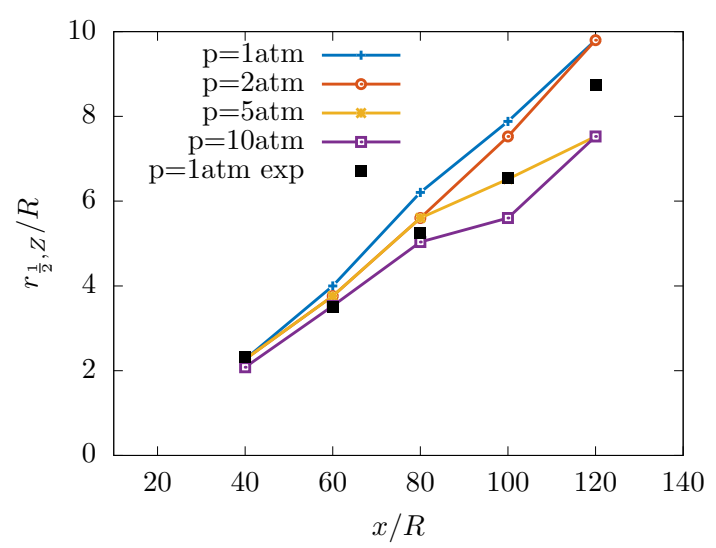

(a)

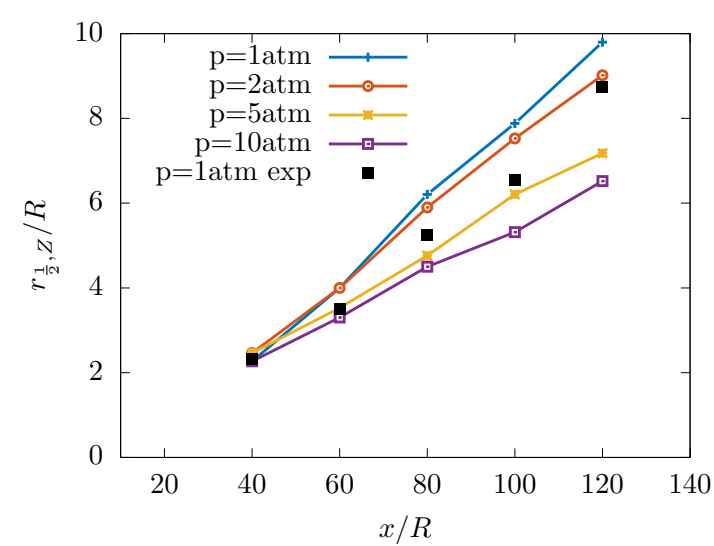

(b)

Figure 26: Nondimensional half-width of mixture fraction $r_{\frac{1}{2}, Z} / R$ as a function of the nondimensional axial coordinate $x / R$ for: series A, series B, and atmospheric reference Sandia/ETH-Zurich Flame A experiment.

Flame A at atmospheric conditions are reported in the same figures. As far as $r_{\frac{1}{2}, Z}$ is concerned, all the jet flames show a monotonic incresing trend, with a good agreement with the atmospheric experimental data, while pressure effects are visible in terms of jet spread angle decrease for both series A and B. This is in agreement with the earlier observation - a narrowing of the flame brush 


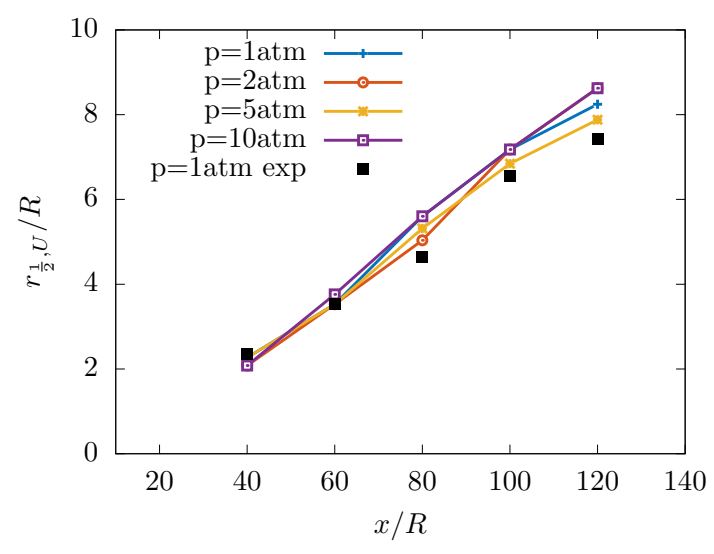

(a) Series-A constant Reynolds number

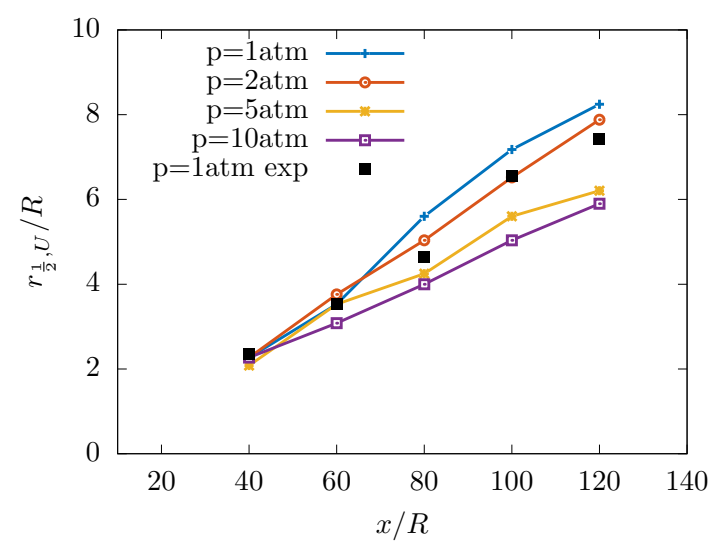

(b) Series-B constant inflow velocity

Figure 27: Nondimensional half-width of axial velocity $r_{1 / 2, U} / R$ as a function of the nondimensional axial coordinate $x / R$ for: series A, series B, and atmospheric reference Sandia/ETH-Zurich Flame A experiment.

with a pressure increase, for both series A and B.

The nondimensional axial velocity half-width $r_{\frac{1}{2}, U} / R$ for both series $\mathrm{A}$ and $\mathrm{B}$ is reported in Figures 27a and 27b respectively, as a function of the non-dimensional axial distance $x / R$. Also in this case, the corresponding experimental measurements of Sandia/ETH-Zurich Flame A at atmospheric conditions are reported as a reference. Similarly to what observed for the mixture fraction half width, $r_{\frac{1}{2}, U} / R$ exhibits a close-to-linear correlation with the nondimensional axial location $x / R$, with a good agreement between numerical and experimental results for the atmospheric case. In this case though, the sensitivity to pressure of the axial velocity half-width is negligible for the series A, as shown in Fig 27a This being coherent with all the findings on the mixture fraction and axial velocity sensitivity on pressure when Reynolds is kept constant. On the other hand, the velocity-based jet spread angle, defined as $S_{U}=d r_{\frac{1}{2}, U} / d x$, decreases with pressure when the inflow velocity is kept constant, as shown in Fig.27b. Also this result is consistent with the previous findings, i.e. longer jet flames when pressure increases in series B. The velocity-based spread angle is reported in table 1 for both series A and B, the spread angle is found to monotonically decrease from $S_{U}=0.078$ to 0.046 going from 1 to 10 atm in series B. 


\begin{tabular}{|l|l|l|l|l|}
\hline Series & \multicolumn{2}{|c|}{$\mathrm{A}$} & \multicolumn{2}{c|}{$\mathrm{B}$} \\
\hline $\mathrm{p}$ [atm] & $S_{U}$ & $x_{0, U}$ & $S_{U}$ & $x_{0, U}$ \\
\hline 1 & 0.078 & 11.2 & 0.078 & 11.2 \\
\hline 2 & 0.083 & 16.7 & 0.069 & 7.1 \\
\hline 5 & 0.073 & 8.9 & 0.052 & -3.9 \\
\hline 10 & 0.082 & 13.9 & 0.046 & -8.1 \\
\hline
\end{tabular}

Table 1: Velocity-based spread-angle $S_{U}=\frac{d r_{\frac{1}{2}, U}}{d x}$ and virtual origin $x_{0, U}$ sensitivity to pressure for series A and B.

\section{Conclusions}

The effects of increasing pressure on a canonical, syngas/air, turbulent, nonpremixed jet flame have been numerically investigated by means of a LES-SLF approach. The applicability of the SLF approach in the context of high pressure has been assessed a priori using an extensive parametric analysis on laminar counterflow flames and CSP/TSR analysis on canonical flame structures. The laminar flame study has shown that an increase in pressure leads to smaller chemical time scales for a fixed scalar dissipation rate, contributing to an increase in the Damkhöler number, justifying the validity of the SLF approach at higher pressures. In addition, the LES-SLF numerical framework has been successfully validated at atmospheric pressure against the Sandia/ETH-Zurich $\mathrm{CO} / \mathrm{H}_{2} / \mathrm{N}_{2}$ experimental database.

Two numerical campaigns, each consisting of three cases with pressure values of 2,5 , and $10 \mathrm{~atm}$ have been carried out in synergy with the current experimental effort on high pressure combustion at KAUST. In the first campaign (series A), the jet Reynolds number has been kept constant by varying the inflow velocity consistently with pressure. In the second campaign (series B), the mean inflow velocity has been kept constant, with a linear increase in jet Reynolds number with pressure.

The simulation results were compared with recent experimental measurements of the same configuration [6], showing good agreement in terms of the $\mathrm{OH}$ layer thickness at different pressure conditions. The insensitivity of the flame position to pressure was also qualitatively in agreement with experimental observations. 
Detailed LES data further revealed the local scalar fields, leading to the findings that both the mean and variance of the scalar dissipation rate decrease with pressure, while the chemical reactivity increases with pressure, thereby pushing the local turbulent flames away from extinction conditions. This suggests that turbulent jet flame characteristics at elevated pressures are most likely to retain the laminar flame structure.

However, the implication of elevated pressure on pollutant formation, such as soot or $\mathrm{NO}_{x}$, needs further investigation.

The information obtained from the two numerical campaigns serve as an archival benchmark to be referenced for future work when more experimental measurements become available, and are expected to be useful in the development of a consistent experimental framework for canonical flames characterization at elevated pressures.

\section{Acknowledgments}

The authors acknowledge the support of Italian Ministry of University and Research (MIUR), as well as that provided by KAUST 1975-03 CCF Subaward Agreement. The second author was partly supported by Advanced Research Center Program (2013R1A5A1073861) and Basic Science Research Program (2017R1A2B4003327) through the National Research Foundation of Korea.

\section{References}

[1] R. S. Barlow, G. J. Fiechtner, C. D. Carter, and M. Flury. Sandia ETH-Zurich CO/H2/N2 Flame Data - Release 1.1. Sandia National Laboratories, pages 1-14, 2002.

[2] R. S. Barlow and J. H. Frank. Effects of turbulence on species mass fractions in methane/air jet flames. In Symposium (International) on Combustion, volume 27, pages 1087-1095, 1998.

[3] R. S. Barlow and J. H. Frank. Piloted CH4/Air Flames C, D, E, and F - Release 2.1. Sandia National Laboratories, pages 1-12, 2007.

[4] R. W. Bilger. Conditional moment closure for turbulent reacting flow. Physics of Fluids, 5(A):436-444, 1993.

[5] W. R. Boyette, T. F. Guiberti, G. Magnotti, and W. L Roberts. Structure of Turbulent Nonpremixed Syngas Flames at High Pressure. Proceedings of the Combustion Institute, paper under review, 2019. 
[6] Wesley R. Boyette, Thibault F. Guiberti, Gaetano Magnotti, and William L. Roberts. Structure of turbulent nonpremixed syngas flames at high pressure. Proceedings of the Combustion Institute, 2018.

[7] Zhi Chen, Shaohong Ruan, and Nedunchezhian Swaminathan. Simulation of turbulent lifted methane jet flames: Effects of air-dilution and transient flame propagation. Combustion and Flame, 162(3):703-716, 2015.

[8] Zhi Chen, Shaohong Ruan, and Nedunchezhian Swaminathan. Large eddy simulation of flame edge evolution in a spark-ignited methane-air jet. Proceedings of the Combustion Institute, 36(2):1645-1652, 2017.

[9] P. P. Ciottoli, R. Malpica Galassi, P. E. Lapenna, G. Leccese, D. Bianchi, F. Nasuti, F. Creta, and M. Valorani. CSP-based chemical kinetics mechanisms simplification strategy for non-premixed combustion: An application to hybrid rocket propulsion. Combustion and Flame, 186:83-93, 2017.

[10] F. Di Sabatino, T. F. Guiberti, W. R. Boyette, W. L. Roberts, J. P. Moeck, and D. A. Lacoste. Effect of pressure on the transfer functions of premixed methane and propane swirl flames. Combustion and Flame, 193:272-282, 2018.

[11] Pascale Domingo, Luc Vervisch, and Denis Veynante. Large-eddy simulation of a lifted methane jet flame in a vitiated coflow. Combustion and Flame, 152(3):415-432, 2008.

[12] E. Giacomazzi, F. R. Picchia, N. Arcidiacono, D. Cecere, F. Donato, and B. Favini. Unsteady simulation of a CO/H2/N2/air turbulent non-premixed flame. Combustion Theory and Modelling, 12(6):1125-1152, 2008.

[13] J. C. Hewson and A. R. Kerstein. Stochastic simulation of transport and chemical kinetics in turbulent CO/H2/N2 flames. Combustion Theory and Modelling, 5(4):669-697, 2001.

[14] Matthias Ihme, Chong M Cha, and Heinz Pitsch. Prediction of local extinction and re-ignition effects in nonpremixed turbulent combustion using a flamelet/progress variable approach. Proceedings of the Combustion Institute, 30(1):793-800, 2005.

[15] Matthias Ihme and Yee Chee See. Prediction of autoignition in a lifted methane/air flame using an unsteady flamelet/progress variable model. Combustion and Flame, 157(10):1850-1862, 2010.

[16] Canny J. A computational approach to edge detection. IEEE Trans Pattern Anal Mach Intell, 8(6):679-98, 1986 Jun.

[17] H. I Joo and Ö. L. Gülder. Soot formation and temperature field structure in co-flow laminar methane-air diffusion flames at pressures from 10 to 60atm. Proceedings of the Combustion Institute, 32(1):769-775, 2009.

[18] A. E. Karataş and Ö. L. Gülder. Soot formation in high pressure laminar diffusion flames. Progress in Energy and Combustion Science, 38(6):818-845, 2012.

[19] R. J. Kee, J. A. Miller, G. H. Evans, and G. Dixon-Lewis. A computational model of the structure and extinction of strained, opposed flow, premixed methane-air flames. Symposium (International) on Combustion, 22(1):1479-1494, 1989.

[20] Konstantin A Kemenov, Haifeng Wang, and Stephen B Pope. Modelling effects of subgrid-scale mixture fraction variance in les of a piloted diffusion flame. Combustion Theory and Modelling, 16(4):611-638, 2012. 
[21] G. Kim, S. Kang, Y. Kim, R. W. Bilger, and M. J. Cleary. Conditional moment closure and transient flamelet modelling for detailed structure and NOx formation characteristics of turbulent nonpremixed jet and recirculating flames. Combustion Theory and Modelling, 11(4):527-552, 2007.

[22] S. Kim, S. Kang, and Y. Kim. Flamelet Modeling for Combustion Processes and NOx Formation in the Turbulent Nonpremixed CO/H2/N2 Jet Flames. Combustion Science and Technology, 168(1):47-83, 2001.

[23] S. K. Kim and Y. Kim. Assessment of the Eulerian particle flamelet model for nonpremixed turbulent jet flames. Combustion and Flame, 154(1-2):232-247, 2008.

[24] S. H. Lam. Using CSP to Understand Complex Chemical Kinetics. Combustion Science and Technology, 89(56):375-404, 1993

[25] Ivan Langella, Zhi X Chen, Nedunchezhian Swaminathan, and Suresh K Sadasivuni. Large-eddy simulation of reacting flows in industrial gas turbine combustor. Journal of Propulsion and Power, 34(5):1269-1284, 2018.

[26] P. E. Lapenna, P. P Ciottoli, and F. Creta. The effect of fuel composition on the non-premixed flame structure of lng/lox mixtures at supercritical pressure. In 54th AIAA Aerospace Sciences Meeting, page 0690, 2016.

[27] P. E. Lapenna, P. P. Ciottoli, and F. Creta. Unsteady non-premixed methane/oxygen flame structures at supercritical pressures. Combustion Science and Technology, 189(12):2056-2082, 2017.

[28] P. E. Lapenna and F. Creta. Direct Numerical Simulation of Transcritical Jets at Moderate Reynolds Number. AIAA Journal, 57(6):2254-2263, 2019.

[29] Pasquale Eduardo Lapenna. Characterization of pseudo-boiling in a transcritical nitrogen jet. Physics of Fluids, 30(7):077106, 2018.

[30] Pasquale Eduardo Lapenna and Francesco Creta. Mixing under transcritical conditions: An a-priori study using direct numerical simulation. The Journal of Supercritical Fluids, 128:263-278, 2017.

[31] Pasquale Eduardo Lapenna, Giuseppe Indelicato, Rachele Lamioni, and Francesco Creta. Modeling the equations of state using a flamelet approach in lre-like conditions. Acta Astronautica, 158:460-469, 2019.

[32] P.E. Lapenna, R. Lamioni, P.P. Ciottoli, and F. Creta. Low-mach number simulations of transcritical flows. AIAA Aerospace Sciences Meeting, 2018, (210059), 2018.

[33] Juan Li, Zhenwei Zhao, Andrei Kazakov, Marcos Chaos, Frederick L Dryer, and James J Scire. A Comprehensive Kinetic Mechanism for $\mathrm{CO}, \mathrm{CH} 2 \mathrm{O}$, and $\mathrm{CH} 3 \mathrm{OH}$ Combustion. International Journal of Chemical Kinetics, 39(3):109-136, 2007.

[34] Juan Li, Zhenwei Zhao, Andrei Kazakov, and Frederick L. Dryer. An updated comprehensive kinetic model of hydrogen combustion. International Journal of Chemical Kinetics, 36(10):566-575, 2004.

[35] S. Liu, J. C. Hewson, J. H. Chen, and H. Pitsch. Effects of strain rate on high-pressure non-premixed n-heptane autoignition in counterflow. Combust. Flame, 137:320-339, 2004.

[36] A. E. Lutz, R. J. Kee, J. F. Grcar, and F. M. Rupley. Oppdif: A fortran program for computing opposed-flow diffusion flames. Technical Report, SAND-96-8243, 1997. 
[37] R. Malpica Galassi, M. Valorani, H. N. Najm, C. Safta, M. Khalil, and P. P. Ciottoli. Chemical model reduction under uncertainty. Combustion and Flame, 179:242-252, 2017.

[38] Riccardo Malpica Galassi, Pietro P. Ciottoli, Subram Mani Sarathy, Hong G. Im, Samuel Paolucci, and Mauro Valorani. Automated chemical kinetic mechanism simplification with minimal user expertise. Combustion and Flame, 197:439-448, 2018.

[39] Dimitrios Manias, Alexandros-Efstathios Tingas, Francisco E Hernández Pérez, Hong G Im, Riccardo Malpica Galassi, Pietro Paolo Ciottoli, and Mauro Valorani. Analysis of hydrogen/air turbulent premixed flames at different karlovitz numbers using computational singular perturbation. In 2018 AIAA Aerospace Sciences Meeting, page $0364,2018$.

[40] Dimitris M. Manias, Efstathios-Al. Tingas, Francisco E. Hernández Pérez, Riccardo Malpica Galassi, Pietro Paolo Ciottoli, Mauro Valorani, and Hong G. Im. Investigation of the turbulent flame structure and topology at different Karlovitz numbers using the tangential stretching rate index. Combustion and Flame, 200:155-167, 2019.

[41] O. Mannaa, M. S. Mansour, W. L. Roberts, and S. H. Chung. Laminar burning velocities at elevated pressures for gasoline and gasoline surrogates associated with RON. Combustion and Flame, 162(6):2311-2321, 2015.

[42] H. Müller, F. Ferraro, and M. Pfitzner. Implementation of a Steady Laminar Flamelet Model for non-premixed combustion in LES and RANS simulations. 8th International OpenFOAM Workshop, (JUNE):11-12, 2013.

[43] N. Peters. Laminar diffusion flamelet models in non-premixed turbulent combustion. Progress in energy and combustion science, 10(3):319-339, 1984.

[44] N. Peters. LAminar Flamelet Concepts in Turbulent Combustion. pages 1231-1250, 1986.

[45] N. Peters. Turbulent Combustion: Cambridge University Press, Cambridge, 0082000.

[46] Charles D Pierce and Parviz Moin. Progress-variable approach for large-eddy simulation of non-premixed turbulent combustion. Journal of fluid Mechanics, 504:73-97, 2004.

[47] H. Pitsch. Large-eddy simulation of turbulent combustion. Annu. Rev. Fluid Mech., 38:453-482, 2006.

[48] H. Pitsch and H. Steiner. Large-eddy simulation of a turbulent piloted methane/air diffusion flame (Sandia flame D). Physics of Fluids, 12(10):2541, 2000.

[49] T. Poinsot and D. Veynante. Theoretical And Numerical Combustion, 3rd edition. [S.1.] T. Poinsot, 2012.

[50] L. Pons, N. Darabiha, and S. Candel. Pressure effects on nonpremixed strained flames. Combustion and Flame, 152(1):218-229, 2008.

[51] S. B. Pope. Turbulent Flows. Cambridge University Press, 2000.

[52] G. Ribert, N. Zong, V. Yang, L. Pons, N. Darabiha, and S. Candel. Counterflow diffusion flames of general fluids: Oxygen/hydrogen mixtures. Combustion and Flame, 154(3):319-330, 2008.

[53] C D Richards and W M Pitts. Global density effects on the self-preservation behaviour of turbulent free jets. $J$. Fluid Mech, 254:417-435, 1993. 
[54] Shaohong Ruan, Nedunchezhian Swaminathan, and Oliver Darbyshire. Modelling of turbulent lifted jet flames using flamelets: a priori assessment and a posteriori validation. Combustion Theory and Modelling, 18(2):295$329,2014$.

[55] C Safta, H N Najm, and O M Knio. \{TChem\} - A Software Toolkit for the Analysis of Complex Kinetic Models. Sandia Report, SAND2011-3(May), 2011.

[56] J. Smagorinsky. General Circulation Experiments With the Primitive Equations. Monthly Weather Review, 91(3):99-164, 1963.

[57] S. A. Steinmetz, T. Fang, and W. L. Roberts. Soot particle size measurements in ethylene diffusion flames at elevated pressures. Combustion and Flame, 169:85-93, 2016.

[58] M. Valorani. Csptk - a software toolkit for the csp and tsr analysis of kinetic models and the simplification and reduction of chemical kinetics mechanisms. the software can be obtained upon request to m.valorani (mauro.valorani@uniroma1.it).2015.

[59] M. Valorani, P. P. Ciottoli, and R. Malpica Galassi. Tangential stretching rate (TSR) analysis of non premixed reactive flows. Proceedings of the Combustion Institute, 36:-, 2017.

[60] M. Valorani, F. Creta, D. A. Goussis, J. C. Lee, and H. N. Najm. An automatic procedure for the simplification of chemical kinetic mechanisms based on csp. Combustion and Flame, 146(1):29-51, 2006.

[61] M. Valorani, S. Paolucci, E. Martelli, T. Grenga, and P. P. Ciottoli. Dynamical system analysis of ignition phenomena using the Tangential Stretching Rate concept. Combustion and Flame, 162(8):2963-2990, 2015.

[62] Mauro Valorani, Pietro Paolo Ciottoli, Riccardo Malpica Galassi, Samuel Paolucci, Temistocle Grenga, and Emanuele Martelli. Enhancements of the G-Scheme Framework. Flow, Turbulence and Combustion, 101(1):1023-1033, 2018.

[63] Mauro Valorani, Samuel Paolucci, Pietro Paolo Ciottoli, and Riccardo Malpica Galassi. Entropy production and timescales. Combustion Theory and Modelling, 21(1):137-157, 2017.

[64] H. G. Weller, G. Tabor, H. Jasak, and C. Fureby. A tensorial approach to computational continuum mechanics using object-oriented techniques. Computers in Physics, 12:620-631, 1998.

[65] X. Zhao, D. C. Haworth, and E. D. Huckaby. Transported PDF Modeling of Nonpremixed Turbulent CO/H 2 /N 2 Jet Flames. Combustion Science and Technology, 184(5):676-693, 2012.

[66] J Zips, C Traxinger, P Breda, and M Pfitzner. Assessment of presumed/transported probability density function methods for rocket combustion simulations. Journal of Propulsion and Power, pages 1-18, 2019.

[67] Julian Zips, Hagen Müller, and Michael Pfitzner. Efficient thermo-chemistry tabulation for non-premixed combustion at high-pressure conditions. Flow, Turbulence and Combustion, 101(3):821-850, 2018. 


\section{Appendix A. Validation of the numerical simulation}

The validation of the numerical predictions is carried out by comparing with the published Sandia/ETH-Zurich experimental database [1]. The inflow velocity profile employed as inflow condition corresponds to a fully developed turbulent pipe, i.e. $U(r)=U_{\max } r^{1 / n}$, where $r$ is the pipe non-dimensional inner radius $y /(D / 2)$, while $U_{\max }$ is the maximum velocity value. The value of $n$ corresponding to the Reynolds number of interest is 6.6 , while $U_{\max }$ is $105.4 \mathrm{~m} / \mathrm{s}$. In accordance with the experiments, the turbulence intensity is chosen to be $0.1 U_{\max }$ as white noise added to the mean inflow velocity, while the coflow velocity is prescribed at $0.6 \mathrm{~m} / \mathrm{s}$ to match the experimental value.

The axial distribution of the mean and root-mean-square (RMS) values of axial velocity are compared to the Sandia/ETH experimental results in Fig. A.28a, showing that the LES results lie within the uncertainty of the experiments in the entire axial range, with a slight discrepancy of both mean and RMS values in the axial location at approximately 10 diameters.

A good agreement between numerical and experimental results is found for the axial distributions of mean and RMS values of mixture fraction and temperature, as shown in Figures A.28cA.28d and A.28e A.28f respectively, highlighting that the numerical approach is capable of accurately reproducing both mixing and chemical processes. A slight over-prediction of the RMS of velocity, temperature and mixture fraction is observed at $x / D=20$. As illustrated in Fig. A.28f. both the numerical and experimental distributions of the temperature RMS exhibit two peaks: the first at $x / D=20$ is associated with small scales oscillations associated to turbulent mixing, while the second, located at $x / D=50$,is due to the alternating detachment of hot products emanating from the jet, possibly due do entrainment effects. In addition, this dynamics is visible in all simulations as shown in the instantaneous temperature fields in Figs.7 and 7, both in terms of temperature profiles and stoichiometric mixture fraction iso-contour line.

A good agreement between the LES and the Sandia/ETH experimental results is observed in terms of radial distribution of temperature and mixture fraction mean and RMS, as seen from Figures A.29 and A.30, respectively. From Figures A.29a and A.29c it appears that at $x / D=30$

the mean stoichiometric mixture fraction $\left\langle\widetilde{Z_{\text {stoi }}}\right\rangle=0.295$ is located at the radial distance $y / D \approx 2$, 


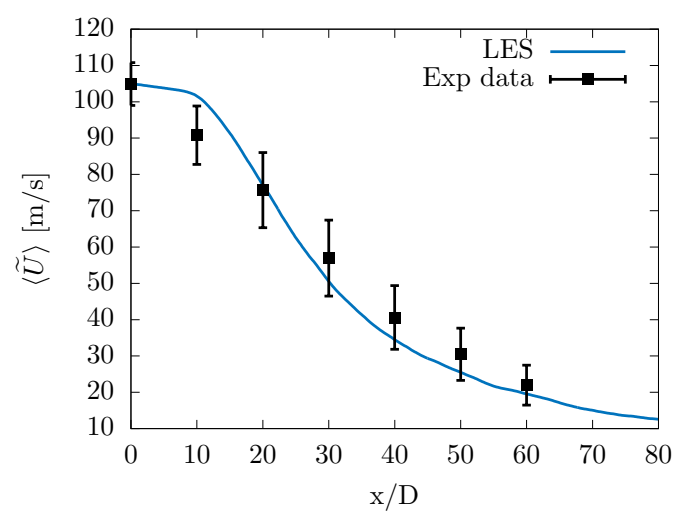

(a) Axial velocity mean

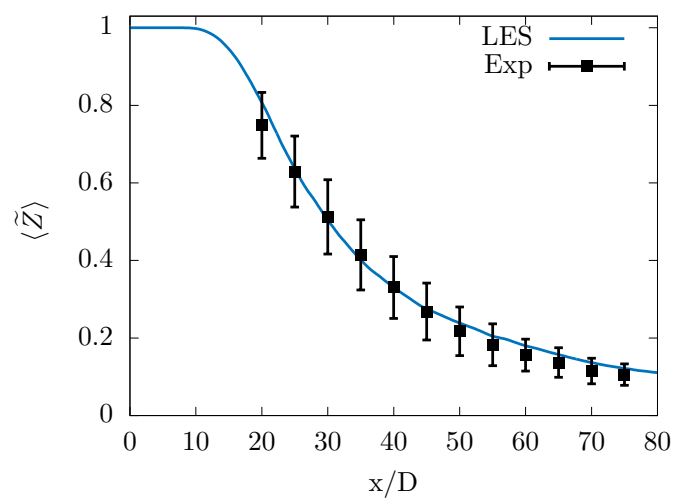

(c) Mixture fraction mean

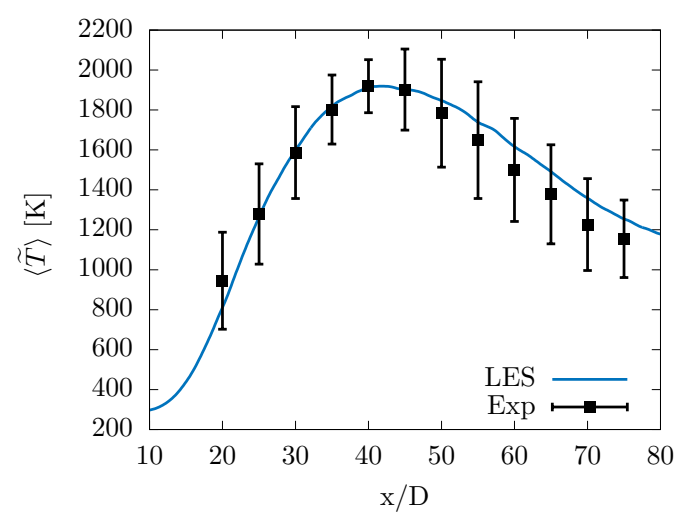

(e) Temperature mean

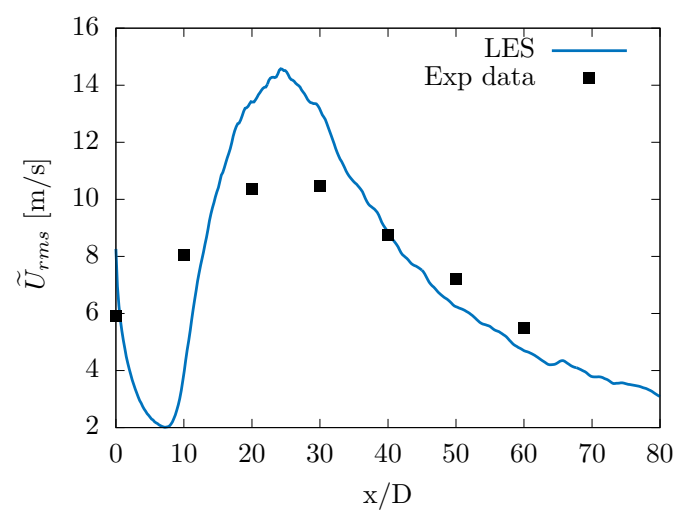

(b) Axial velocity RMS

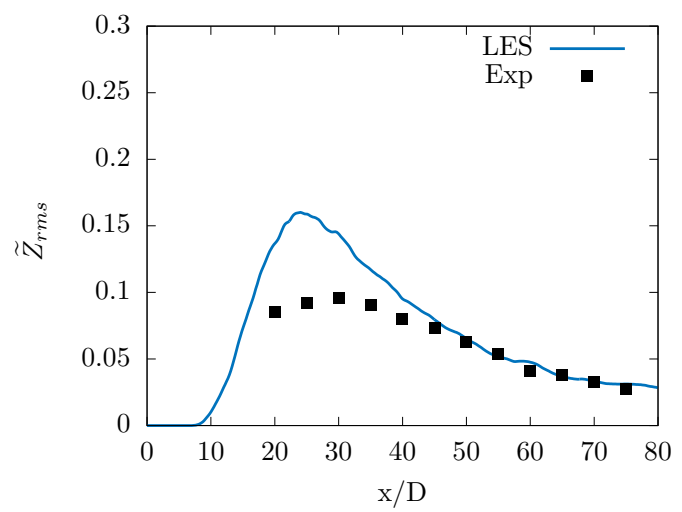

(d) Mixture fraction RMS

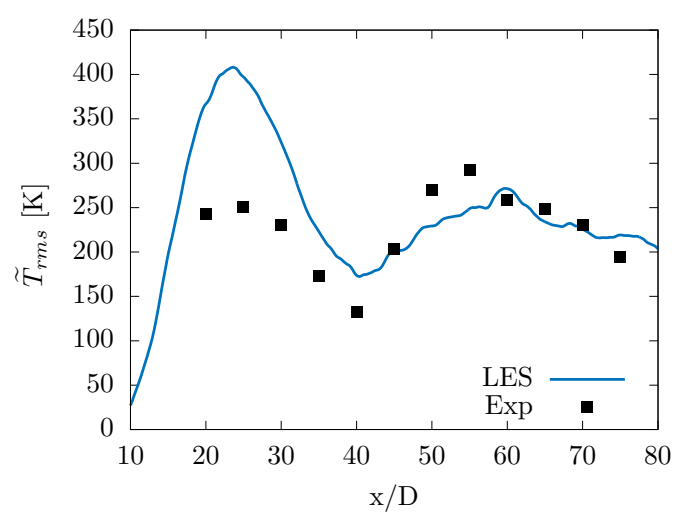

(f) Temperature RMS

Figure A.28: Numerical results validation against Sandia/ETH-Zurich Flame A atmospheric experimental data. Axial profiles of axial velocity, mixture fraction and temperature mean and RMS values. 


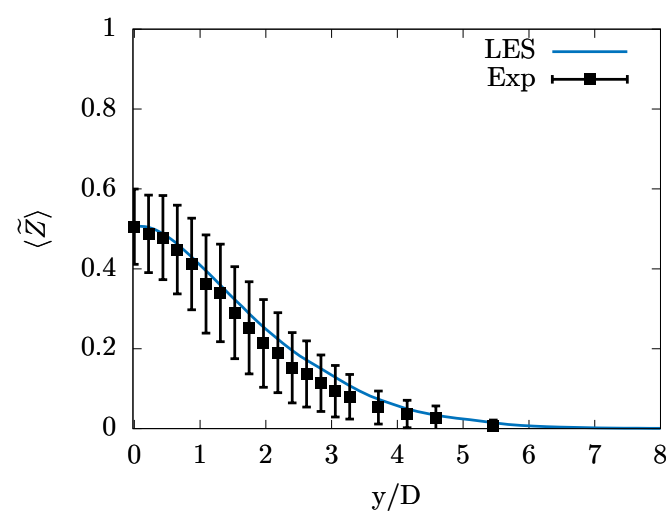

(a) Mean value at $\mathrm{x} / \mathrm{D}=30$

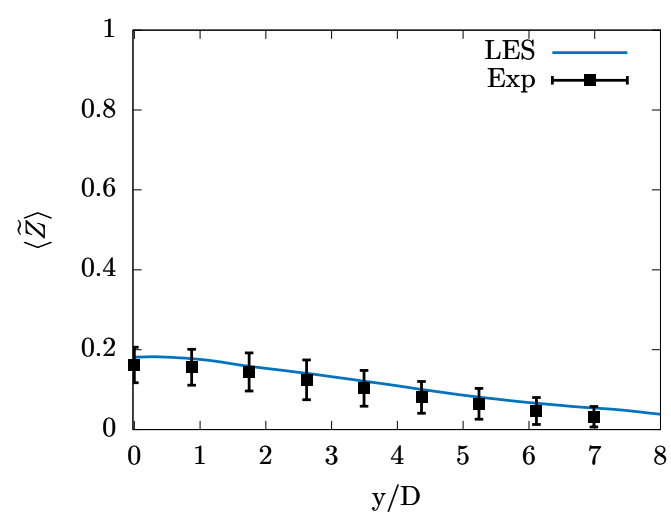

(c) Mean value at $\mathrm{x} / \mathrm{D}=60$

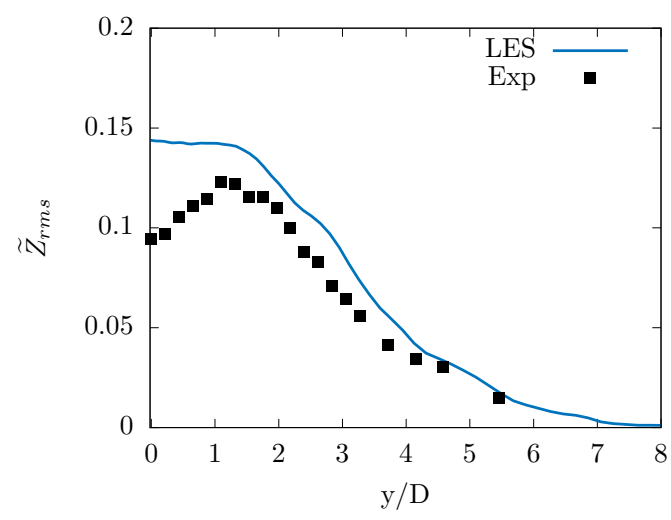

(b) RMS at $x / D=30$

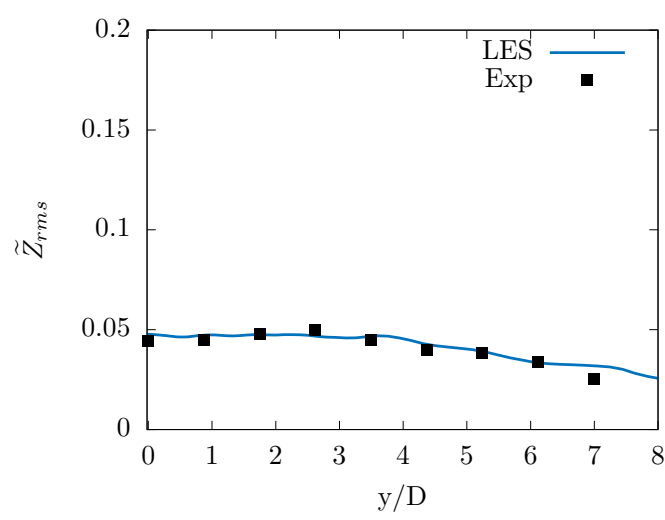

(d) RMS at $x / D=60$

Figure A.29: Numerical results validation against Sandia/ETH-Zurich Flame A atmospheric experimental data. Radial profiles of mixture fraction mean and RMS values at normalized distances from the inlet, $\mathrm{x} / \mathrm{D}=30$ and 60 .

while the maximum mean mixture fraction value at $x / D=60$ is 0.2 , indicating that at this axial distance the mixture is always highly diluted. These observations are in accordance with the mean temperature radial distribution at $x / D=30$ and $x / D=60$, shown in Figures A.30a and A.30c. The RMS fluctuations of the mixture fraction drops significantly from $x / D=30$ to $x / D=60$, as shown in Figures A.29b and A.29d. Similarly, the RMS of temperature fluctuations, shown in Figures A.30b and A.30d, also show a decayed and flatter radial profiles from $x / D=30$ to $x / D=60$. Radial profiles of the mean values of various major and minor species are plotted in Fig. A.31 for axial distances of $x / D=20,40$ and 60 . The agreement between the numerical results and the experimental measurements is consistent with that for the mixture fraction and temperature 


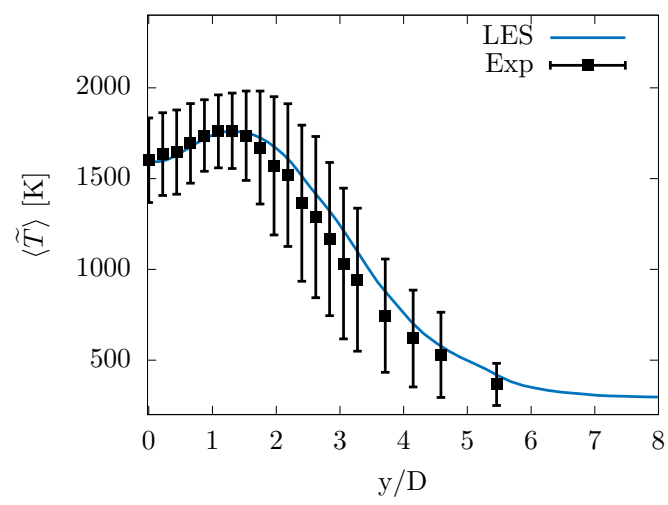

(a) Mean value at $\mathrm{x} / \mathrm{D}=30$

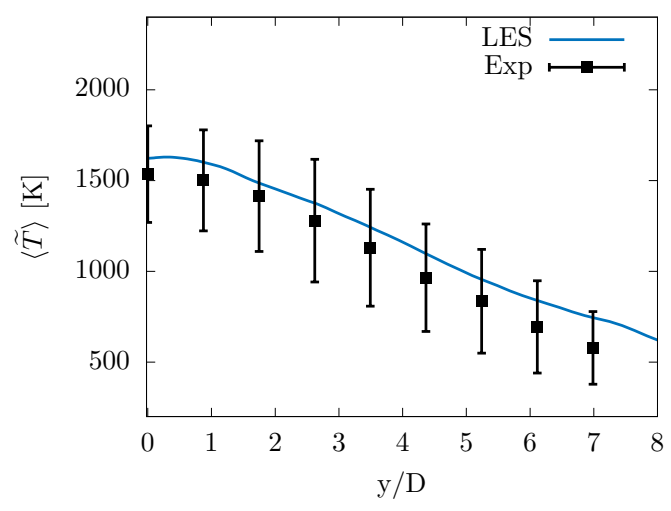

(c) Mean value at $\mathrm{x} / \mathrm{D}=60$

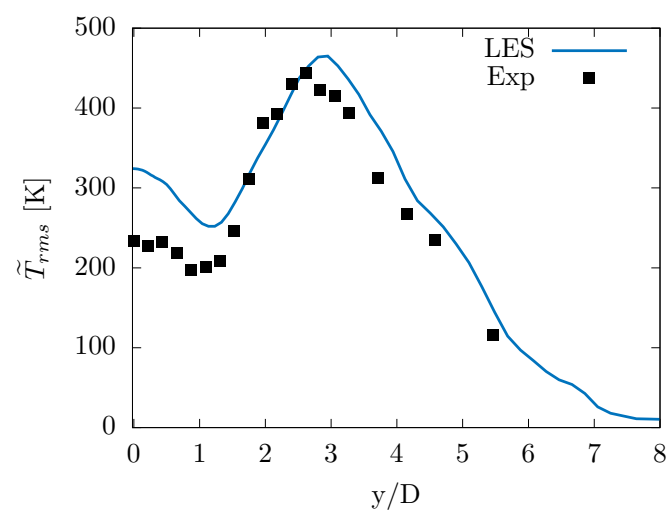

(b) RMS at $x / D=30$

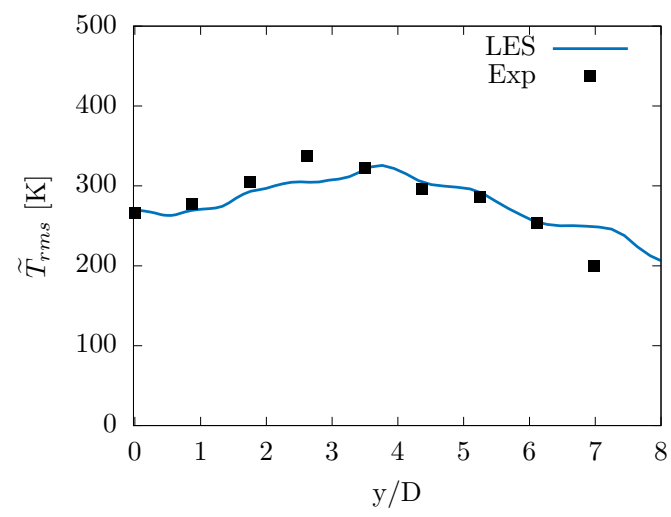

(d) RMS at $x / D=60$

Figure A.30: Numerical results (LES) validation against Sandia/ETH-Zurich Flame A atmospheric experimental data (Exp). Radial profiles of temperature mean and RMS values at normalized distances from the injector of $\mathrm{x} / \mathrm{D}=30$ and 60.

distribution. Some notable discrepancies are observed in the $\mathrm{OH}$ profiles, especially with a underprediction at $x / D=40$, although it is still within the experimental uncertainties. Overall, we conclude that the present LES numerical simulations successfully reproduced the experimental results. 


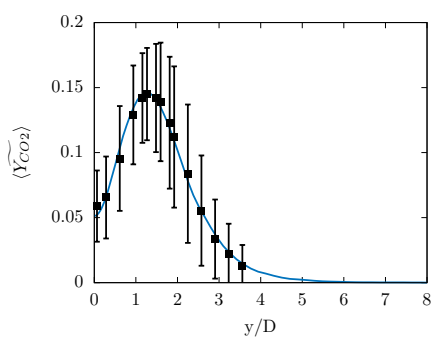

(a) $x / D=20$

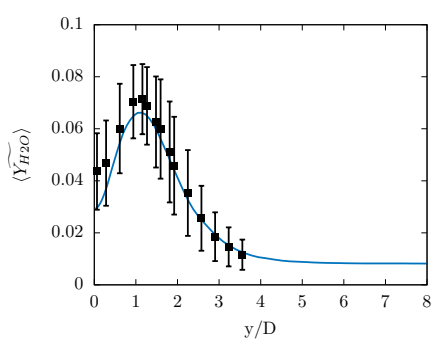

(d) $x / D=20$

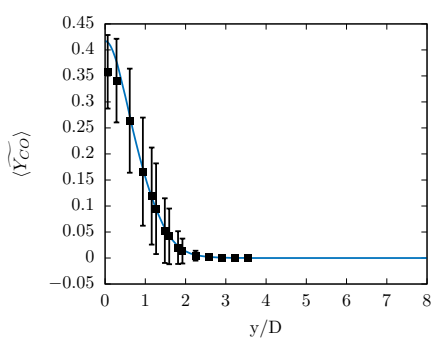

(g) $x / D=20$

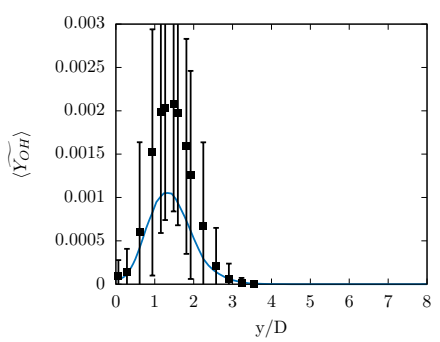

(j) $x / D=20$

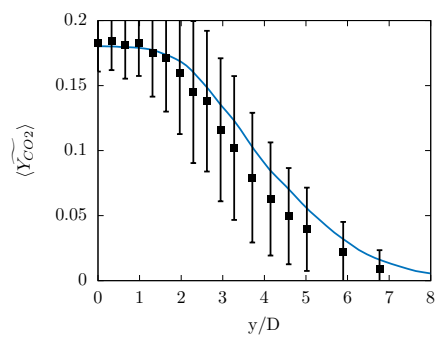

(b) $x / D=40$

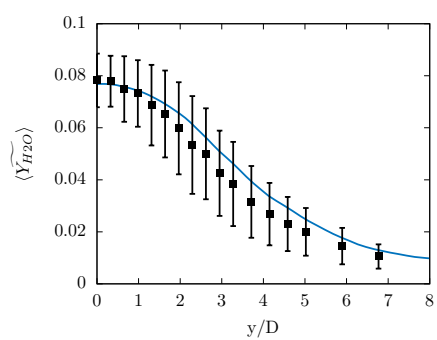

(e) $x / D=40$

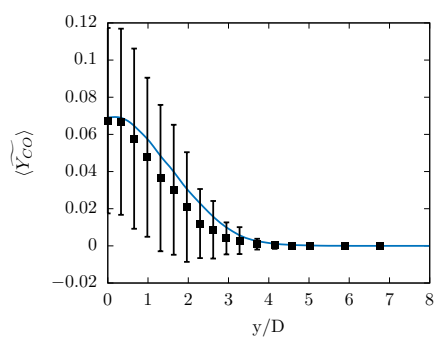

(h) $x / D=40$

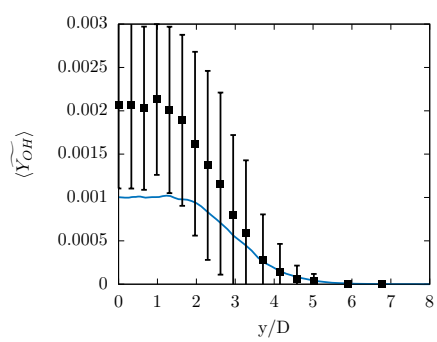

(k) $x / D=40$

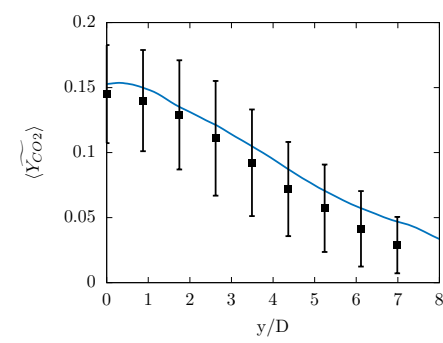

(c) $x / D=60$

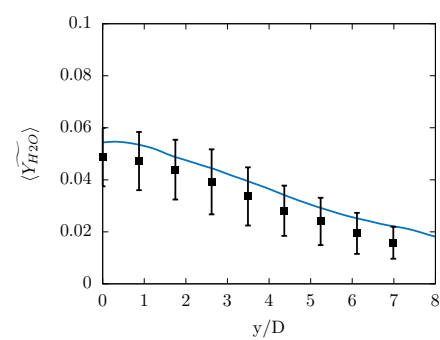

(f) $x / D=60$

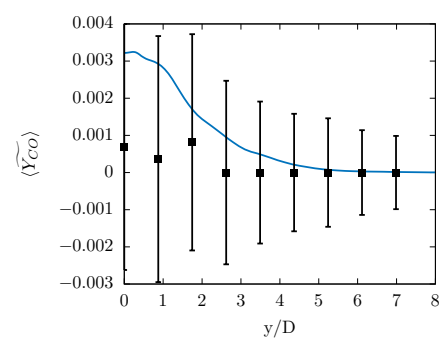

(i) $\mathrm{x} / \mathrm{D}=60$

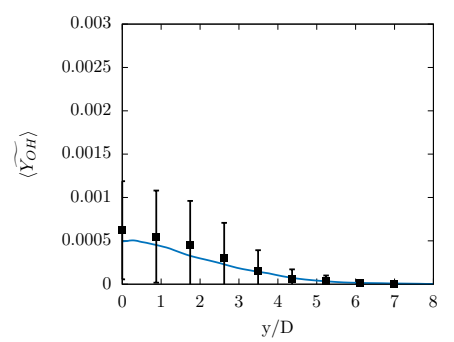

(1) $x / D=60$

Figure A.31: Numerical results (solid line) validation against Sandia/ETH-Zurich Flame A atmospheric experimental data (symbols). Radial profiles of $\mathrm{CO}_{2}, \mathrm{H}_{2} \mathrm{O}, \mathrm{CO}$, and $\mathrm{H}_{2}$ mass fraction mean values at normalized distances from the inlet of $\mathrm{x} / \mathrm{D}=20,40$ and 60 . 